ITEP-TH-30/03

August 22, 2003

\title{
Bihamiltonian structures and quadratic algebras in hydrodynamics and on non-commutative torus
}

\author{
B.Khesin \\ Max Planck Institute of Mathematics, Bonn, Germany \\ University of Toronto, Toronto, Canada \\ e-mail: khesin@math.toronto.edu \\ A.Levin \\ Max Planck Institute of Mathematics, Bonn, Germany \\ Institute of Oceanology, Moscow, Russia \\ e-mail: alevin@wave.sio.rssi.ru \\ M. Olshanetsky \\ Max Planck Institute of Mathematics, Bonn, Germany \\ Institute of Theoretical and Experimental Physics, Moscow, Russia, \\ e-mail: olshanet@gate.itep.ru
}

\begin{abstract}
We demonstrate the common bihamiltonian nature of several integrable systems. The first one is an elliptic rotator that is an integrable Euler-Arnold top on the complex group $\operatorname{GL}(N, \mathbb{C})$ for any $N$, whose inertia ellipsiod is related to a choice of an elliptic curve. Its bihamiltonian structure is provided by the compatible linear and quadratic Poisson brackets, both of which are governed by the Belavin-Drinfeld classical elliptic $r$-matrix. We also generalize this bihamiltonian construction of integrable Euler-Arnold tops to several infinitedimensional groups, appearing as certain large $N$ limits of $\operatorname{GL}(N, \mathbb{C})$. These are the group of a non-commutative torus (NCT) and the group of symplectomorphisms $\operatorname{SDiff}\left(T^{2}\right)$ of the two-dimensional torus. The elliptic rotator on symplectomorphisms gives an elliptic version of an ideal 2D hydrodynamics, which turns out to be an integrable system. In particular, we define the quadratic Poisson algebra on the space of Hamiltonians on $T^{2}$ depending on two irrational numbers. In conclusion, we quantize the infinite-dimensional quadratic Poisson algebra in a fashion similar to the corresponding finite-dimensional case.
\end{abstract}

\section{Contents}

1 Introduction $\quad 2$

2 Main results $\quad 3$

$3 \operatorname{GL}(N, \mathbb{C})$-Elliptic Rotators $\quad 6$

3.1 Lax representation and integrals of motion . . . . . . . . . . . . 6

3.2 The classical $\mathrm{GL}(N, \mathbb{C})$ Feigin-Odesski algebras $\ldots \ldots \ldots \ldots \ldots$

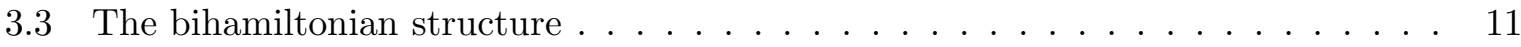


4 Elliptic Rotators on non-commutative torus 13

4.1 The Lax equation and integrals of motion . . . . . . . . . . . . . . . . 13

4.2 Quadratic Poisson algebras and the bihamiltonian structure on NCT . . . . . . 16

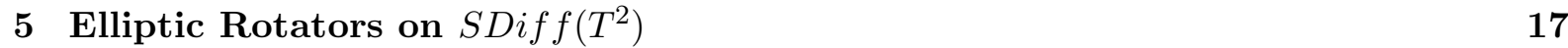

5.1 Description of the hierarchy . . . . . . . . . . . . . . . 17

5.2 Quadratic Poisson algebras, and the bihamiltonian structure on SDiff $\left(T^{2}\right) \ldots \ldots$

5.3 Rational limit of the elliptic hydrodynamics . . . . . . . . . . . . . 20

6 Quantum counterparts 22

$\begin{array}{ll}\text { Acknowledgments } & 25\end{array}$

7 Appendix $\quad 25$

7.1 Appendix A. Elliptic functions. . . . . . . . . . . . . . . . . . . . 25

7.2 Appendix B. Elliptic constants related to $\operatorname{gl}(N, \mathbb{C}) \ldots \ldots \ldots \ldots \ldots$

7.3 Appendix C. Non-commutative torus . . . . . . . . . . . . . . . . 28

\section{Introduction}

One of the most interesting problems in the theory of integrable systems is a description of infinite-dimensional integrable systems with two or more space variables. In this paper we consider integrable hierarchies in $(2+1)$ dimension, which we call elliptic and modified hydrodynamics. These are close relatives of the ideal hydrodynamics on a two-dimensional torus, which is known to be a highly non-integrable system. For instance, even the 4-vortex approximation of the ideal fluid dynamics is non-integrable [1]. We obtain these integrable versions of hydrodynamics starting from finite-dimensional integrable systems whose dimension of the phase space goes to infinity. Our approach is somewhat similar to the derivation of Toda field theory from the open Toda chain. In contrast with the Toda theory, however, we obtain non-local systems.

The classical Euler (or, rather, Helmholtz) equation for the motion of an ideal fluid on the standard two-torus is

$$
\partial_{t} \mathbf{S}=\left\{\mathbf{S}, \Delta^{-1} \mathbf{S}\right\}
$$

where $\mathbf{S}$ is the vorticity function of the fluid flow, \{\} is the Poisson bracket, and $\Delta$ is the Laplace operator on $T^{2}$. The modified and elliptic hydrodynamics are defined on an elliptic curve, i.e., on a torus with a complex structure fixed. The corresponding equations are, respectively,

$$
\partial_{t} \mathbf{S}=\left\{\mathbf{S}, \bar{\partial}^{-2} \mathbf{S}\right\}
$$

and

$$
\partial_{t} \mathbf{S}=\{\mathbf{S}, \wp(\bar{\partial}) \mathbf{S}\}
$$

where $\bar{\partial}$ is the corresponding operator of the complex structure, and $\wp$ is the Weierstrass $\wp$ function. (We postpone the precise description of $\wp(\bar{\partial})$ till Section 5.) Note that to define the Laplace operator one needs to choose a metric, while for the operator $\bar{\partial}$ in the modified hydrodynamics is defined by a complex structure on $T^{2}$.

Such a modification of the fluid inertia operator from the Laplace operator $\Delta=\partial \bar{\partial}$, which depends on a metric on $T^{2}$, to $\bar{\partial}^{2}$ or $\wp(\bar{\partial})^{-1}$, both of which depend on a complex structure on $T^{2}$, brings in the integrability and even the bihamiltonian structure for the systems. We construct an infinite set of involutive integrals of motion with respect to two Poisson brackets. 
One of the brackets is the standard linear Lie-Poisson brackets on the dual space to the algebra of the divergence-free vector fields on $T^{2}$. The other Poisson structure is a quadratic Poisson algebra on Hamiltonians of vector fields. These two brackets are compatible and governed by the same classical $r$-matrix. Furthermore, we describe a recursion procedure for constructing the sequence of Hamiltonians for this linear-quadratic bihamiltonian structure of the hierarchy of the elliptic hydrodynamics, which thereby exhibits "the strongest form of integrability."

We come to this construction through the non-commutative deformation of $T^{2}$ to the noncommutative torus (NCT). The non-commutative deformation of the Lie algebra of vector fields is isomorphic to the Lie algebra of NCT. The latter is a special large $N$ limit of $\operatorname{gl}(N, \mathbb{C})$. We start with the elliptic rotators on $\operatorname{GL}(N, \mathbb{C})[2,3]$ and develop their bihamiltonian structure based on the Belavin-Drinfeld classical $r$-matrix [4]. The corresponding quadratic Poisson algebra is the classical limit of the Feigin-Odesski algebra [5]. We describe natural extensions of the elliptic rotators to the infinite-dimensional groups, which preserve their main properties. This brings us to bihamiltonian systems and integrable hierarchies on the NCT, which are of interest by themselves.

As a byproduct, we also describe a quantization of the quadratic Poisson algebra on NCT. In this way we obtain an infinite-dimensional associative algebra with quadratic relations depending on the complex structure on $T^{2}$ and the Planck constant $\hbar \in T^{2}$.

Finally, the commutative ("dispersionless") limit of the elliptic rotators on NCT leads to the desirable bihamiltonian hierarchies of the elliptic and modified hydrodynamics.

\section{Main results}

1. The general setup $[6,7]$

Let $G$ be a Lie group and $\mathfrak{g}$ its Lie algebra. Consider an invertible linear operator $\mathbf{J}$ that maps the coalgebra $\mathfrak{g}^{*}$ to $\mathfrak{g}$. Its inverse operator $\mathbf{J}^{-1}$ is called the inertia tensor. The Euler-Arnold top corresponding to the group $G$ is the Hamiltonian system on $\mathfrak{g}^{*}$ with respect to the linear Lie-Poisson brackets on $\mathfrak{g}^{*}$ and the Hamiltonian function given by the quadratic form

$$
H=-\frac{1}{2}\langle\mathbf{S}, \mathbf{J}(\mathbf{S})\rangle, \quad \mathbf{S} \in \mathfrak{g}^{*},
$$

where $\langle$,$\rangle stands for the pairing between \mathfrak{g}$ and $\mathfrak{g}^{*}$. Namely, the variation $\nabla H$ can be regarded as an element of $\mathfrak{g}$ and the corresponding Hamiltonian equation of motion is as follows:

$$
\partial_{t} \mathbf{S}=\{H, \mathbf{S}\}:=\operatorname{ad}_{\nabla H}^{*} \mathbf{S} .
$$

Recall that the Lie-Poisson brackets are degenerate on $\mathfrak{g}^{*}$ and their symplectic leaves are coadjoint orbits of $G$. To descend to a particular coadjoint orbit $\mathcal{O}$ one should fix the values of Casimirs for the linear bracket.

For some special choices of $\mathbf{J}$ the system becomes completely integrable. Some of the examples are Manakov's tops on $S O(N)$ [8], their limit $N \rightarrow \infty$ found by Ward [9], the Korteweg-de Vries equation [10, 11], as well as the Camassa-Holm and Hunter-Saxton equations on the Virasoro group [12]. (In the infinite-dimensional case, the invertibility of $\mathbf{J}$ is understood as $\mathrm{Ker} \mathbf{J}=0$.)

2. Elliptic Rotators on $\operatorname{GL}(N, \mathbb{C})$

The elliptic rotator $(\mathrm{ER})$ on $\mathrm{GL}(N, \mathbb{C})$ is an integrable Euler-Arnold top on this group, whose inertia operator is constructed with the help of the Weierstrass $\wp$-function of an auxilliary 
elliptic curve, see Ref. $[2,3,13]$. Namely, let $T_{\alpha}, \alpha=\left(\alpha_{1}, \alpha_{2}\right)$ be the basis of $\operatorname{sl}(N, \mathbb{C})(B .4$, $\alpha_{j}=(0,1, \ldots, N-1), \alpha \neq(0,0)$. The structure constants in this basis are

$$
\mathbf{C}_{\theta}(\alpha, \beta)=\frac{1}{\pi \theta} \sin \pi \theta(\alpha \times \beta), \quad \theta=k / N,
$$

where $1 \leq k<N$ and $k, N$ are coprime, see (B.8). For $\mathbf{S}=\sum_{\alpha} S_{-\alpha} T_{\alpha} \in \operatorname{sl}(N, \mathbb{C})^{*}$ the linear Poisson brackets assume the form

$$
\left\{S_{\alpha}, S_{\beta}\right\}=\mathbf{C}_{\theta}(\alpha, \beta) S_{\alpha+\beta}
$$

Let $\wp(x ; \tau)$ be the Weierstrass function on the elliptic curve $E_{\tau}=\mathbb{C} /(\mathbb{Z}+\tau \mathbb{Z})$. Consider its values $\wp_{\theta}(\alpha)=\wp\left(\left(\alpha_{1}+\alpha_{2} \tau\right) \theta ; \tau\right)$ on the lattice parametrized by $\alpha$, the labels of the $\operatorname{sl}(N, \mathbb{C})$-basis. The inverse inertia operator is defined as

$$
\mathbf{J}: S_{\alpha} \rightarrow J_{\alpha} S_{\alpha}, \quad J_{\alpha}=\wp_{\theta}(\alpha),
$$

It was proved in Ref.[2] that the equations of motion

$$
\partial_{t} S_{\alpha}=\sum_{\gamma} S_{\alpha-\gamma \wp_{\theta}(\gamma) S_{\gamma}}
$$

defined by the Hamiltonian $H=-\frac{1}{2}\langle\mathbf{S}, \mathbf{J}(\mathbf{S})\rangle=2 \pi^{2} \theta^{2} \operatorname{tr}(\mathbf{S} \cdot \mathbf{J}(\mathbf{S}))$ and the brackets (2.1), have the Lax representation with the Lax operator $L^{r o t}(\mathbf{S}, z) \in \operatorname{sl}(N, \mathbb{C})$ depending on the spectral parameter $z \in E_{\tau}$. The traces $\operatorname{tr}\left(L^{r o t}(\mathbf{S}, z)\right)^{k}, k=2, \ldots, N$ being expanded in the basis of elliptic functions on $E_{\tau}$ produce the involutive integrals of motion and the hamiltonian $H$ is among them.

We call the commuting flows defined by these integrals the ER hierarchy.

Let $r(z), z \in E_{\tau}$ be the Belavin-Drinfeld classical elliptic r-matrix, see [13, 4] and Section 3 below. The main result, which we will also generalize to the infinite-dimensional situation, can be formulated as follows.

Theorem 2.1 i) In terms of the r-matrix, the linear brackets (2.1) can be written in the form

$$
\left\{L_{1}^{r o t}(z), L_{2}^{r o t}(w)\right\}_{1}=\left[r(z-w), L_{1}^{r o t}(z)+L_{2}^{r o t}(w)\right] .
$$

ii) Consider the phase space extended by a new variable $S_{0}$, which has zero bracket with all the rest. The same $r$-matrix defines the quadratic Poisson algebra $P_{N, \theta, \tau}$ related to $\operatorname{GL}(N, \mathbb{C})(3.19)$, (3.20):

$$
\left\{L_{1}(z), L_{2}(w)\right\}_{2}=\left[r(z-w), L_{1}(z) \otimes L_{2}(w)\right], \text { where } L(z)=S_{0} I d+L^{r o t}(z) .
$$

iii) The above two brackets are compatible, i.e. any linear combination of them is a Poisson bracket.

iv) There exists a sequence of integrals of motion in involution with respect to each of the two brackets $\left\{h_{j}, h_{k}\right\}_{1,2}=0$ (here the lower indices refer to the linear and the quadratic brackets respectively). They provide the bihamiltonian structure of the elliptic rotator (ER) hierarchy

$$
\left\{h_{j+1}, \mathbf{S}\right\}_{2}=-\left\{h_{j}, \mathbf{S}\right\}_{1}
$$

The first two statements of Theorem for $\operatorname{GL}(N, \mathbb{C})$ are well known $[5,14]$. Apparently, the bihamiltonian structure of the $\mathrm{GL}_{N}$ elliptic rotators is new, and it gives the following 
Corollary 2.1 The Casimirs with respect to one of the brackets generate non-trivial dynamics with respect to the other.

In particular, the functional $S_{0}$, being the Casimir element of the linear brackets (2.1), leads to the equations

$$
\partial_{t} \mathbf{S}=\left\{S_{0}, \mathbf{S}\right\}_{2}
$$

that coincide with (2.3).

It turns out that Theorem 2.1 also holds in the infinite-dimensional situation presented below.

\section{Elliptic Rotators on the Non-commutative Torus}

Consider the $\sin$-algebra $\sin _{\theta}$, called also the Lie algebra of the non-commutative torus (NCT). The Poisson brackets on the Lie coalgebra $\sin _{\theta}^{*}$ of the NCT has the form (2.1) with irrational number $0 \leq \theta<1$ and the basis $T_{\alpha}=$ const $\cdot \exp \left(2 \pi i \alpha_{1} x_{1}\right) * \exp \left(2 \pi i \alpha_{2} x_{2}\right)$ is parameterized by the infinite lattice $\alpha \in \mathbb{Z} \oplus \mathbb{Z}$. Here $\exp \left(2 \pi i x_{1}\right), \exp \left(2 \pi i x_{2}\right)$ are the generators of the NCT and $*$ is the Moyal multiplication (see Appendix C). On the NCT we introduce the complex structure $\bar{\partial}_{\epsilon, \tau}$ depending on $\tau(\operatorname{Im} \tau>0)$, and two real numbers $\epsilon=\left(\epsilon_{1}, \epsilon_{2}\right)$, such that $\theta \epsilon_{a}$ are irrational and $0<\theta \epsilon_{a} \leq 1$ (see (4.3)). The inverse inertia operator $\mathbf{J}$ is the pseudo-differential operator

$$
\mathbf{J}(\mathbf{S})(x)=\wp\left(\theta \bar{\partial}_{\epsilon, \tau}\right) \mathbf{S}(x),
$$

where now $\mathbf{S}(x)=\sum_{\alpha} S_{-\alpha} T_{\alpha}(x)$. The equation of motion in the form of the Moyal brackets has the form

$$
\partial_{t} \mathbf{S}=\left\{\mathbf{S}, \wp\left(\theta \bar{\partial}_{\epsilon, \tau}\right) \mathbf{S}\right\}^{\theta}
$$

where \{\}$^{\theta}$ is the Lie-Poisson bracket on $\sin _{\theta}^{*}$. In Proposition 3.1 we find the Lax form of (2.7). We construct the classical $r$-matrix (4.11) and prove the counterpart of the Theorem. The quadratic Poisson algebra $P_{\theta, \epsilon, \tau}$ (4.19), (4.20) on the NCT gives rise to the infinite bihamiltonian hierarchy, where the equations (2.7) correspond to the quadratic Hamiltonian functional with respect to the linear brackets. At the same time the equations (2.7) can be interpreted as the Hamiltonian equations (2.6) in the quadratic Poisson algebra $P_{\theta, \epsilon, \tau}$ with a linear Hamiltonian. For $\mathrm{SL}(2)$ this representation was found in [15] and for general case in [18].

\section{Elliptic Rotators on $\operatorname{SDiff}\left(T^{2}\right)$}

In the limit $\theta \rightarrow 0$ the Lie algebra of the NCT becomes isomorphic to the Poisson algebra $\mathcal{A}$ of smooth functions on $T^{2}$ modulo constants $\operatorname{Ham}\left(T^{2}\right) \sim C^{\infty}\left(T^{2}\right) / \mathbb{C}$. The algebra of Hamiltonians can be also described by the corresponding Hamiltonian (or, divergence-free) vector fields on the torus. More precisely, the Lie algebra $S V$ ect $\left(T^{2}\right)$ of divergence-free vector fields on $T^{2}$ is the (universal) cocentral extension of $\operatorname{Ham}\left(T^{2}\right)$, defined by the exact sequence

$$
0 \rightarrow \operatorname{Ham}\left(T^{2}\right) \rightarrow \operatorname{SVect}\left(T^{2}\right) \rightarrow \mathbb{C}^{2} \rightarrow 0,
$$

where the image of $S \operatorname{Vect}\left(T^{2}\right)$ in $\mathbb{C}^{2}$ is generated by the two fluxes $\epsilon_{1} \partial_{1}, \epsilon_{2} \partial_{2}$. For $\psi \in \operatorname{Ham}\left(T^{2}\right)$ we have

$$
V_{1}(\psi)=-\frac{1}{4 \pi^{2}} \partial_{2} \psi, \quad V_{2}(\psi)=\frac{1}{4 \pi^{2}} \partial_{1} \psi,
$$

and $V_{\left\{\psi, \psi^{\prime}\right\}}=\left[V(\psi), V(\psi)^{\prime}\right]$. We construct the elliptic rotator on the Lie group SDiff $\left(T^{2}\right)$ of area-preserving diffeomorphisms, corresponding to the Lie algebra $S V \operatorname{ect}\left(T^{2}\right)$.

Consider the dual space of linear functionals $\operatorname{Ham}\left(T^{2}\right)^{*}$ in the Fourier basis

$$
\left\{\mathbf{e}(\alpha \cdot x):=\exp 2 \pi i(\alpha \cdot x) \mid\left(x=\left(x_{1}, x_{2}\right),(\alpha \cdot x)=\alpha_{1} x_{1}+\alpha_{2} x_{2}, \alpha_{j} \in \mathbb{Z}\right\},\right.
$$




$$
\mathbf{S}=\sum_{\alpha} S_{\alpha} \mathbf{e}(-(\alpha \cdot x)) \in \operatorname{Ham}\left(T^{2}\right)^{*} .
$$

The Poisson structure on $\mathcal{A}^{*}$ assumes the form (cf. (2.1))

$$
\left\{S_{\alpha}, S_{\beta}\right\}_{1}=(\alpha \times \beta) S_{\alpha+\beta} .
$$

This Poisson structure is degenerate and has an infinite set of Casimirs

$$
C_{k}=\int_{T^{2}} \mathbf{S}^{k}, \text { where } k=2,3, \ldots
$$

On a coadjoint orbit $\mathcal{O} \subset \operatorname{Ham}\left(T^{2}\right)^{*}$ of $\operatorname{SDiff}\left(T^{2}\right)$ the brackets are non-degenerate and values of the Casimirs are fixed.

Define the operator $\mathbf{J}$ (after the rescaling) as the pseudo-differential operator $\wp\left(\bar{\partial}_{\epsilon, \tau}\right)$, such that the Hamiltonian has the form

$$
H=-\frac{1}{2} \int_{T^{2}} \mathbf{S} \cdot \wp\left(\bar{\partial}_{\epsilon, \tau}\right) \mathbf{S} .
$$

We prove that the corresponding Hamiltonian system admits an infinite set of commuting integrals, see Section 5.

Recall that these elliptic rotators are parameterized, in particular, by the auxiliary elliptic curve. Consider the simplest version of the ER, corresponding to the rational degeneration of the elliptic curve $E_{\tau}$. In the limit the (inverse) inertia operator becomes $\mathbf{J}=\bar{\partial}_{\tau}^{-2}$ and the limiting Hamiltonian (2.8) takes the form

$$
H=-\frac{1}{2} \int_{T^{2}} \mathbf{S} \cdot \bar{\partial}_{\tau}^{-2} \mathbf{S}
$$

see (5.23) and [15].

It is interesting to compare this with the Euler equation for an ideal fluid on a torus, where the inertia operator is the Laplacian (i.e., $\mathbf{J}=\Delta^{-1}=(\bar{\partial} \partial)^{-1}$ ), and the Hamiltonian is

$$
H=-\frac{1}{2} \int_{T^{2}} \mathbf{S} \cdot \Delta^{-1} \mathbf{S}
$$

where $\mathbf{S}$ plays the role of the vorticity [7]. While the Euler hydrodynamics equation is highly non-integrable, the modification of the (inverse) inertia operator from $\bar{\partial} \partial$ to $\bar{\partial}_{\tau}^{2}$ leads to an integrable hierarchy. We call the rational limit the modified hydrodynamics on $T^{2}$. (In a sense, these systems are similar in spirit to the Etingof-Frenkel current algebras [16] on $T^{2}$, which make use of the complex structure on the elliptic curve to construct a central extension of smooth currents on the torus.)

Finally, we prove the Theorem 2.1 for the generic systems of the elliptic hydrodynamics (2.8). In particular, the quadratic Poisson algebra $\mathcal{P}_{0, \tau}(5.27)$, (5.28) on $T^{2}$, along with the linear Poisson bracket, provide the bihamiltonian structure of the hierarchies of the elliptic and modified hydrodynamics.

\section{$3 \operatorname{GL}(N, \mathbb{C})$-Elliptic Rotators}

\subsection{Lax representation and integrals of motion}

1. The system description

The elliptic $\operatorname{GL}(N, \mathbb{C})$-rotator is defined on the dual spaces of $\operatorname{gl}(N, \mathbb{C})$ and $\operatorname{sl}(N, \mathbb{C})$. Let $\mathbf{S}=$ 
$\sum_{\alpha} S_{-\alpha} T_{\alpha}$, where $T_{\alpha}$ is the basis of $\operatorname{sl}(N, \mathbb{C})$, see (B.4). Then the Poisson structure on the dual $\operatorname{space} \operatorname{sl}(N, \mathbb{C})^{*}$ is given by the linear Lie-Poisson brackets $($ B.8)

$$
\left\{S_{\alpha}, S_{\beta}\right\}_{1}=\mathbf{C}_{\theta}(\alpha, \beta) S_{\alpha+\beta},
$$

where $\mathbf{C}_{\theta}(\alpha, \beta)$ are the structure constants of $\operatorname{sl}(N, \mathbb{C})($ B.8). The Hamiltonian has the form

$$
H^{r o t}=2 \pi^{2} \theta^{2} \operatorname{tr}(\mathbf{S} \cdot \mathbf{J}(\mathbf{S})) \equiv-\frac{1}{2} \sum_{\gamma} S_{\gamma} \wp_{\gamma} S_{-\gamma} .
$$

It defines the equations of motion

$$
\partial_{t} \mathbf{S}=[\mathbf{J}(\mathbf{S}), \mathbf{S}]
$$

Let $\mathbf{J}: S_{\alpha} \rightarrow J_{\alpha} S_{\alpha}$, be the (inverse) inertia operator with $J_{\alpha}=\wp_{\theta}(\alpha)$, where $\wp_{\theta}(\alpha)$ are the values of the Weierstrass $\wp$-function and defined by (B.11). Then (3.3) assumes the form

$$
\partial_{t} S_{\alpha}=\sum_{\gamma} S_{\alpha-\gamma} S_{\gamma \wp_{\theta}}(\gamma) \mathbf{C}_{\theta}(\gamma, \alpha)
$$

2. The linear brackets and $r$-matrix

Proposition 3.1 (cf.[2]) The equations of motion (3.2) have the Lax form

$$
\partial_{t} L^{r o t}(z)=\left[L^{r o t}(z), M(z)\right],
$$

where

$$
\begin{aligned}
L^{r o t} & =-\sum_{\alpha} S_{\alpha} \varphi_{\theta}(\alpha, z) T_{\alpha}, \\
M & =-\sum_{\alpha} S_{\alpha} f_{\theta}(\alpha, z) T_{\alpha},
\end{aligned}
$$

and the functions $\varphi_{\theta}, f_{\theta}$ are given by (B.13), (B.14).

Proof.

Substituting (3.6) and (3.7) in the Lax equation we obtain

$$
\partial_{t} S_{\alpha} \varphi_{\theta}(\alpha, z)=\sum_{\gamma} S_{\alpha-\gamma} S_{\gamma}\left(\varphi_{\theta}(\alpha-\gamma, z) f_{\theta}(\gamma, z)-\varphi_{\theta}(\gamma, z) f_{\theta}(\alpha-\gamma, z)\right) .
$$

Now, using the explicit expressions (B.13) and (B.14) for $\varphi_{\theta}$ and $f_{\theta}$ respectively, as well as the Calogero functional equation (A.22), we come to

$$
\partial_{t} S_{\alpha}=\sum_{\gamma} S_{\alpha-\gamma} S_{\gamma} \varphi_{\theta}(\alpha, z)\left(\wp_{\theta}(\alpha-\gamma)-\wp_{\theta}(\gamma)\right)
$$

which coincides with (3.4).

The Lie-Poisson brackets (3.1) admit the following r-matrix description [4, 13, 14, 17]. Define the classical $r$-matrix by

$$
r_{N, \theta, \tau}(z-w)=\sum_{\gamma} \varphi_{\theta}(\gamma, z-w) T_{\gamma} \otimes T_{-\gamma},
$$

where $\varphi_{\theta}$ is defined by (B.13). 
Lemma 3.1 [14]. The r-matrix (3.8) satisfies the classical Yang-Baxter equation

$$
\begin{gathered}
{\left[r_{N, \theta, \tau}(z-w), r_{N, \theta, \tau}(z)\right]+\left[r_{N, \theta, \tau}(z-w), r_{N, \theta, \tau}(w)\right]} \\
+\left[r_{N, \theta, \tau}(z), r_{N, \theta, \tau}(w)\right]=0 .
\end{gathered}
$$

Proof.

We reduce the YB equation (3.9) to the following functional equation

$$
\varphi_{\theta}(\gamma, z-w) \varphi_{\theta}(\alpha, z)-\varphi_{\theta}(\alpha+\gamma, z-w) \varphi_{\theta}(\alpha, w)+\varphi_{\theta}(\alpha+\gamma, z) \varphi_{\theta}(-\gamma, w)=0,
$$

by using the commutation relations $\operatorname{in} \operatorname{sl}(N, \mathbb{C})($ B.7). It easy to see that it can be rewritten for the functions $\phi_{\theta}$ (B.12), since all three terms have the common exponent $\mathbf{e}_{\theta}\left(\left(\gamma_{2}+\alpha_{2}\right) z-\gamma_{2} w\right)$. Now, it coincides with the Fay identity (A.21), where we put

$$
u_{1}=(\gamma+\alpha) \theta, \quad u_{2}=-\gamma \theta, \quad z_{1}=z, \quad z_{2}=w .
$$

Proposition 3.2 In terms of the Lax operator (3.7) the brackets (3.1) are equivalent to the following relation for the Lax operator

$$
\begin{gathered}
\left\{L_{1}^{r o t}(z), L_{2}^{r o t}(w)\right\}_{1}=\left[r_{N, \theta, \tau}(z-w), L^{r o t}(z) \otimes I d+I d \otimes L^{r o t}(w)\right], \\
L_{1}=L \otimes I d, \quad L_{2}=I d \otimes L .
\end{gathered}
$$

Proof.

To prove (3.10) we rewrite it in the basis $T_{\alpha} \otimes T_{\beta}$ :

$$
\begin{gathered}
\left\{S_{-\alpha}, S_{-\beta}\right\} \varphi_{\theta}(\alpha, z) \varphi_{\theta}(\beta, w) \\
=S_{-\alpha-\beta} C_{\theta}(\alpha, \beta)\left(\varphi_{\theta}(-\beta, z-w) \varphi_{\theta}(\alpha+\beta, z)-\varphi_{\theta}(\alpha, z-w) \varphi_{\theta}(\alpha+\beta, w)\right) .
\end{gathered}
$$

The same Fay identity (A.21) implies that

$$
\varphi_{\theta}(\alpha, z) \varphi_{\theta}(\beta, w)=\varphi_{\theta}(-\beta, z-w) \varphi_{\theta}(\alpha+\beta, z)-\varphi_{\theta}(\alpha, z-w) \varphi_{\theta}(\alpha+\beta, w) .
$$

Thus we come to (3.1).

3. The hierarchy of the Lax equations

The Lax operator $L^{\text {rot }}$ (3.6) has the following properties:

i) $L^{\text {rot }}$ is an $\operatorname{sl}(N, \mathbb{C})$-valued meromorphic function on $E_{\tau}$ with a simple pole at the origin satisfying

$$
\left.\operatorname{Res} L^{r o t}(z)\right|_{z=0}=\sum_{\alpha} S_{-\alpha} T_{\alpha} .
$$

ii) $L^{r o t}$ satisfies the quasi-periodicity conditions

$$
L^{r o t}(z+1)=Q L^{r o t}(z) Q^{-1}, \quad L^{r o t}(z+\tau)=\Lambda L^{r o t}(z) \Lambda^{-1} .
$$

These properties imply that $\operatorname{Tr}(L(z))^{k}$ are doubly periodic functions with the poles up to the order $k$. Thereby, they can be expanded in the basis of the Weierstrass function and its derivatives

$$
\operatorname{tr}\left(L^{r o t}(z)\right)^{k}=I_{0, k}+I_{2, k} \wp(z)+\ldots+I_{k, k} \wp^{(k-2)}(z) .
$$

In particular, in this way we obtain the Hamiltonian

$$
\operatorname{tr}\left(L^{r o t}(z)\right)^{2}=I_{0,2}+I_{2,2} \wp(z), \quad I_{0,2}=2\left(\frac{i}{2 \pi \theta}\right)^{2} H, \quad I_{2,2}=\operatorname{tr} \mathbf{S}^{2} .
$$


Proposition 3.3 The coefficients $I_{s, k}$ are in involution with respect to the linear bracket (3.1)

$$
\left\{I_{s, k}, I_{m, j}\right\}_{1}=0 .
$$

Proof

We have

$$
\left\{\operatorname{tr}\left(L_{1}^{r o t}(z)\right)^{k}, \operatorname{tr}\left(L_{2}^{r o t}(w)\right)^{j}\right\}_{1}=\operatorname{tr}\left\{\left(L_{1}^{r o t}(z)\right)^{k},\left(L_{2}^{r o t}(w)\right)^{j}\right\}_{1} .
$$

Then, it follows from (3.10) that these functionals Poisson commute. Using the expansion (3.11) we arrive at the involutivity of the coefficients (3.12).

In particular, all functions $I_{s, k}$ Poisson commute with the Hamiltonian $H$ (3.2). Therefore, they play the role of conservation laws of elliptic rotator hierarchy on $\operatorname{GL}(N, \mathbb{C})$. We have a tower of $\frac{N(N+1)}{2}$ independent integrals of motion

$$
\begin{array}{ccccc}
I_{0,2} & I_{2,2} & & & \\
I_{0,3} & I_{2,3} & I_{3,3} & & \\
\ldots & \ldots & \ldots & \ldots & \\
I_{0, n} & I_{2, N} & \ldots & \ldots & I_{N, N}
\end{array}
$$

Note that $I_{k, k}, k=0,2,3 \ldots, N$ are the Casimirs corresponding to the coadjoint orbit

$$
\mathcal{R}^{r o t}=\left\{\mathbf{S} \in \operatorname{gl}(N, \mathbb{C}), \quad \mathbf{S}=g^{-1} \mathbf{S}^{(0)} g\right\} .
$$

The conservation laws $I_{s, k}$ generate commuting flows on $\mathcal{R}^{\text {rot }}$

$$
\partial_{s, k} \mathbf{S}=\left\{I_{s, k}, \mathbf{S}\right\}_{1}, \quad\left(\partial_{s, k}:=\partial_{t_{s, k}}\right),
$$

that we call $E R_{N}$ hierarchy.

In what follows we will need another set of the conservation laws coming from the coefficients of the spectral curve

$$
\mathcal{C}: F(\lambda, z) \equiv \operatorname{det}\left(\lambda \cdot I d+L^{r o t}(z)\right)=0 .
$$

Here

$$
\begin{gathered}
F(\lambda, z)=\lambda^{N}+\lambda^{N-2} a_{2}(z)+\ldots+a_{N}(z), \\
a_{k}(z)=J_{0 k}+J_{2 k} \wp(z)+\ldots+J_{k k} \wp^{(k-2)}(z) .
\end{gathered}
$$

Since $a_{k}(z)$ and $\operatorname{Tr}(L(z))^{k}$ are related by the Newton formula, one can recursively represent $J_{s, k}$ as polynomials of $I_{m, j}, j \leq k, m \leq j$

$$
J_{s, k}=\sum a_{\vec{l}, \vec{m}, \vec{j}}^{s, k} \prod_{\sum j_{i} m_{i}=k, l_{i} \leq j_{i}}\left(I_{l_{i}, j_{i}}\right)^{m_{i}}, \quad\left(\vec{l}=\left(l_{1}, l_{2}, \ldots\right)\right) .
$$

For example,

$$
\begin{gathered}
J_{s, 2}=-I_{s, 2}, \quad(s=0,2) ; \quad J_{s, 3}=-I_{s, 3}, \quad(s=0,2,3) ; \\
J_{0,4}=\frac{3}{2} I_{0,4}-I_{0,2}^{2}-\frac{g_{2}}{12} I_{2,2}^{2}, \quad\left(g_{2}=60 \sum_{m, n}^{\prime}(m+n \tau)^{-4}\right) ; \\
J_{2,4}=\frac{3}{2} I_{2,4}-2 I_{0,2} I_{2,2} ; \quad J_{3,4}=\frac{3}{2} I_{3,4} \quad J_{4,4}=\frac{3}{2} I_{4,4}-I_{2,2}^{2},
\end{gathered}
$$

where in the second line we have exploited the relation $12 \wp^{2}=2 \wp^{\prime \prime}+g_{2}$. 


\subsection{The classical $\mathrm{GL}(N, \mathbb{C})$ Feigin-Odesski algebras}

Here we consider the Feigin-Odesski Poisson brackets related to $\operatorname{GL}(N, \mathbb{C})$. In notations of Ref. [5] it is the classical limit of the quadratic associative algebra $Q_{N^{2}, k}$.

It turns out that the same $r$-matrix (3.8) defines a quadratic Poisson algebra. We start with the Lax operator $L^{r o t}$ (3.6). Modify it in the following way

$$
L(z)=-S_{0} I d+L^{r o t}(z),
$$

where $S_{0}$ commute with $S_{\alpha}$ with respect to the linear brackets (3.1). Define the brackets between the entries of $L$ as follows

$$
\left\{L_{1}(z), L_{2}(w)\right\}_{2}=\left[r_{N, \theta, \tau}(z-w), L_{1}(z) \otimes L_{2}(w)\right],
$$

(see Ref. $[13,14]$ ). These brackets are Poisson, since the Jacobi identity is provided by the classical Yang-Baxter equation (3.9). A finite-dimensional quadratic Poisson algebra is extracted from (3.18) by eliminating the dependence on the spectral parameters $z, w$.

Proposition 3.4 The quadratic Poisson algebra on $\mathrm{gl}(N, \mathbb{C})^{*}$ has the form

$$
\begin{gathered}
\left\{S_{\alpha}, S_{0}\right\}_{2}=\sum_{\gamma \neq \alpha} S_{\alpha-\gamma} S_{\gamma}\left(\wp_{\theta}(\gamma)-\wp_{\theta}(\alpha-\gamma)\right) \mathbf{C}_{\theta}(\alpha, \gamma), \\
\left\{S_{\alpha}, S_{\beta}\right\}_{2}=S_{0} S_{\alpha+\beta} \mathbf{C}_{\theta}(\alpha, \beta)+\sum_{\gamma \neq \alpha,-\beta} S_{\alpha-\gamma} S_{\beta+\gamma} \mathbf{f}_{\theta}(\alpha, \beta, \gamma) \mathbf{C}_{\theta}(\gamma, \alpha-\beta),
\end{gathered}
$$

where $\mathbf{C}_{\theta}(\alpha, \beta)$ are the $\operatorname{sl}(N, \mathbb{C})$ structure constants (B.8),

$$
\begin{gathered}
\mathbf{f}_{\theta}(\alpha, \beta, \gamma)=\zeta_{\theta}(\gamma)+\zeta_{\theta}(\beta-\alpha+\gamma)-\zeta_{\theta}(\beta+\gamma)+\zeta_{\theta}(\alpha-\gamma) \\
=-\frac{\vartheta^{\prime}(0) \vartheta_{\theta}(\alpha) \vartheta_{\theta}(\beta) \vartheta_{\theta}(\beta-\alpha+2 \gamma)}{\vartheta_{\theta}(\alpha-\gamma) \vartheta_{\theta}(\beta+\gamma) \vartheta_{\theta}(\beta-\alpha+\gamma) \vartheta_{\theta}(\gamma)},
\end{gathered}
$$

and $\vartheta_{\theta}, \zeta_{\theta}$ are the zeta constants (B.10), (B.11).

Proof.

The equation (3.18) yields the following form of the brackets

$$
\left\{S_{a}, S_{b}\right\} \varphi_{\theta}(a, z) \varphi_{\theta}(b, w)=\sum_{\gamma} S_{a-\gamma} S_{b+\gamma} \varphi_{\theta}(\gamma, z-w) \varphi_{\theta}(a-\gamma, z) \varphi_{\theta}(b+\gamma, w) \mathbf{C}_{\theta}(\gamma, a-b) .
$$

Note first, that the common exponents in the expressions of the functions $\varphi_{\theta}$ (B.13) coincide in the left and the right sides. This allows one to pass to the functions $\phi_{\theta}$ and in this way to use the Fay-type identities. We rewrite the last expression in the form

$$
\begin{gathered}
\left\{S_{a}, S_{b}\right\} \phi_{\theta}(z) \phi_{\theta}(w)=\sum_{\gamma} S_{a-\gamma} S_{b+\gamma} \mathbf{C}_{\theta}(\gamma, a-b) \\
\times\left(\phi_{\theta}(\gamma, z-w) \phi_{\theta}(a-\gamma, z) \phi_{\theta}(b+\gamma, w)-\phi_{\theta}(a-b-\gamma, z-w) \phi_{\theta}(b+\gamma, z) \phi_{\theta}(a-\gamma, w)\right) .
\end{gathered}
$$

Consider first the case $b=0$. Then we can apply (A.30) for $u=\left(a_{1}+a_{2} \tau\right) \theta, v=\left(\gamma_{1}+\gamma_{2}\right) \theta$. It immediately leads us to (3.19). If $a, b \neq 0$ we have the terms of two types. For $\gamma \neq a,-b$ one uses (A.27), (A.28) and finds the second term in the right side of (3.20). If $\gamma=a$ or $\gamma-=b$ one 
should take into account that $\phi_{\theta}(0, z)=1$ and then use the Fay identity (A.21). This gives us the first term. The identity (3.21) follows from (A.29).

We denote by $P_{N, \theta, \tau}$ the quadratic Poisson algebra (3.19), (3.20). Recall that $\tau$ is the modular parameter of the auxiliary curve $E_{\tau}$ and $\theta=k / N$. The algebra $P_{2, \frac{1}{2}, \tau}$ is the classical Sklyanin algebra [14]. In this case the r.h.s. of (3.20) contains only the first term.

As for the linear brackets, there exist $N$ Casimirs $C_{0}^{(2)}, C_{2}^{(2)}, \ldots, C_{N}^{(2)}$ of the algebra $P_{N, \theta, \tau}$ [18]. They can be read off from the expansion of the spectral curve (3.13) in the basis of elliptic functions

$$
\operatorname{det}\left(S_{0} \cdot I d+L^{r o t}(z)\right)=C_{0}^{(2)}+\sum_{k=2}^{N} C_{k}^{(2)} \wp^{(k-2)}(z) .
$$

It follows from $(3.13),(3.14)$ and $(3.17)$ that they are linear combinations of the integrals $J_{s, k}$

$$
\begin{gathered}
C_{0}^{(2)}=S_{0}^{N}+\sum_{m=2}^{N} S_{0}^{N-m} J_{0, m}, \\
C_{k}^{(2)}=\sum_{m=0}^{N-k} S_{0}^{N-k-m} J_{k, k+m}, \quad(k=2, \ldots, N) .
\end{gathered}
$$

\subsection{The bihamiltonian structure}

Two Poisson structures are called compatible (or, form a Poisson pair) if their linear combinations are Poisson structures as well.

Proposition 3.5 The linear and quadratic Poisson brackets on $\operatorname{gl}(N, \mathbb{C})$ are compatible.

Proof

Let us replace $S_{0} \rightarrow S_{0}+\lambda$ in the quadratic brackets (3.19), (3.20). Then (3.19) does not change, while (3.20) acquires an additional linear term. In this way we define a one-parameter family of the brackets

$$
\{\mathbf{S}, \mathbf{S}\}_{\lambda}:=\{\mathbf{S}, \mathbf{S}\}_{2}+\lambda\{\mathbf{S}, \mathbf{S}\}_{1}
$$

where $\{\mathbf{S}, \mathbf{S}\}_{1}$ denote the linear brackets (3.1). Therefore, the linear combination $\{\mathbf{S}, \mathbf{S}\}_{\lambda}$ of two brackets can be obtained from the quadratic bracket by a simple shift of $S_{0}$. The result of the shift, of course, still satisfies the Jacobi identity, and hence it is a Poisson bracket for any $\lambda$.

We denote this family of the quadratic Poisson algebras by $P_{N, \theta, \tau, \lambda}$. The algebras are isomorphic for different $\lambda$ and degenerate to the linear Poisson algebra $\operatorname{gl}(N, \mathbb{C})^{*}$ as $\lambda \rightarrow \infty$.

Consider the Casimir functions of the Poisson algebra $P_{N, \theta, \tau, \lambda}$

$$
h_{k}(\lambda)=C_{k}^{(2)}\left(S_{0}+\lambda\right) \quad(k=0,2, \ldots, N) .
$$

It follows from (3.24) and (3.25) that $h_{k}(\lambda)$ are polynomials in $\lambda$

$$
h_{k}(\lambda)=\sum_{m=0}^{N-k} h_{m, k} \lambda^{m}
$$


The coefficients are defined as

$$
h_{a, k}=\frac{1}{a !} \partial_{\lambda}^{(a)} C_{k}^{(2)}\left(S_{0}+\lambda\right)_{\lambda=0}
$$

It implies that

$$
\begin{gathered}
h_{a, 0}=\frac{N !}{(N-a) ! a !} S_{0}^{N-a}+\sum_{m=2}^{N-a} \frac{(N-m) !}{(N-m-a) ! a !} S_{0}^{N-m-a} J_{0, m}, \\
h_{a, k}=\sum_{m=0}^{N-k-a} \frac{(N-k-m) !}{(N-k-m-a) ! a !} S_{0}^{N-k-m-a} J_{k, k+m},(k=2, \ldots, N) .
\end{gathered}
$$

In particular,

$$
h_{N-1,0}=N S_{0}, \quad h_{N-2,0}=\frac{N(N-1)}{2} S_{0}^{2}+J_{0,2} .
$$

Conversely, one can express $J_{m, k+m}$ as a linear combination of $h_{N-m-s, m}, s=0, \ldots, k$ from the relations (3.28) and (3.29). For example,

$$
\begin{gathered}
J_{j, j}=h_{N-j, j}, \quad J_{j, j+1}=h_{N-j-1, j}-(N-j) S_{0} h_{N-j, j}, \\
J_{j, j+2}=h_{N-j-2, j}-(N-j) S_{0} h_{n-j-1, j}+\frac{(N-j)(N-j-1)}{2} S_{0}^{2} h_{N-j, j} .
\end{gathered}
$$

We arrange the quantities $h_{m, k}$ in the triangular tableau

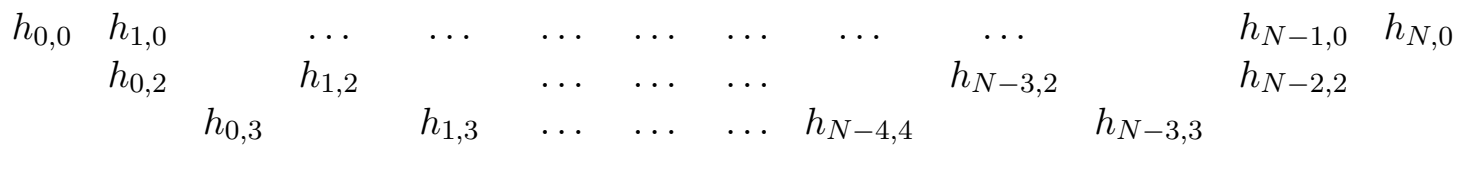

$$
\begin{aligned}
& h_{0, N-1} \quad h_{1, N-1} \\
& h_{0, N}
\end{aligned}
$$

Note, that the second line corresponding to $h_{s, 1}$ is absent since $h_{1}(\lambda)=0$. The left side of the triangle contains the Casimirs $h_{0, j}=C_{j}^{(2)}(3.24)$, (3.25) of $P_{N, \theta, \tau}$, while the right side represents the Casimirs $h_{N-j, j}=J_{j, j}$ of the linear brackets on $\operatorname{gl}(N, \mathbb{C})^{*}$.

The remarkable property of the quantities $h_{a, k}$ is that they are in involution with respect to both the linear and quadratic brackets:

$$
\left\{h_{a, k}, h_{b, j}\right\}_{1,2}=0,
$$

see, e.g., [19]. This fact follows from the identities

$$
\left\{h_{k}(\lambda), h_{j}(\lambda)\right\}_{\lambda}=0
$$

held for each $\lambda$.

Proposition 3.6 The integrals $h_{a, k}$ provide the recurrence relation for the elliptic rotator hierarchy $E R_{N}$

$$
\left\{h_{a, k}, \mathbf{S}\right\}_{1}=-\left\{h_{a+1, k}, \mathbf{S}\right\}_{2} .
$$


Proof.

Since $h_{k}(\lambda)$ are the Casimirs, we have $\left\{h_{k}(\lambda), \mathbf{S}\right\}_{\lambda}=0$. Substitute the representation (3.27) into this equation. Then the recurrence relation (3.33) comes from the coefficient in front of $\lambda^{a}$.

In this way one can start with the Casimirs of the linear brackets and produce a non-trivial dynamical system using the quadratic brackets

$$
\left\{J_{k, k}, \mathbf{S}\right\}_{2}=-\left\{J_{k, k+1}, \mathbf{S}\right\}_{1},
$$

(see (3.30)). In particular, the flow (3.4) corresponding to $H=I_{0,2}$ can be represented by the quadratic brackets with $S_{0}$ :

$$
\partial_{t} \mathbf{S}=\left\{S_{0}, \mathbf{S}\right\}_{2} .
$$

Alternatively, one can start with the Hamiltonians in the left side of the table (3.31) and the linear brackets.

This allows us to conclude the proof of Theorem 2.1 for the hierarchy ER E $_{N}$. Namely, Propositions $3.2,3.4,3.5$, and 3.6 are equivalent, respectively, to i), ii), iii), and iv).

\section{Elliptic Rotators on non-commutative torus}

\subsection{The Lax equation and integrals of motion}

\section{The system description}

The elliptic rotator on the non-commutative torus $\mathcal{A}_{\theta}$ is a generalization of the elliptic rotator on $\operatorname{GL}(N, \mathbb{C})$. We consider the Euler-Arnold top on the group $S I N_{\theta}$ of the NCT algebra $\mathcal{A}_{\theta}$ (Appendix C). The Lie coalgebra $\sin _{\theta}^{*}$ is equipped with the linear Poisson brackets

$$
\left\{S_{\alpha}, S_{\beta}\right\}_{1}=\mathbf{C}_{\theta}(\alpha, \beta) S_{\alpha+\beta}, \quad\left(\alpha \in \tilde{\mathbb{Z}}^{(2)}\right),
$$

where $\mathbf{S}=\sum S_{-\alpha} T_{\alpha}$ and

$$
\tilde{\mathbb{Z}}^{(2)}=\left\{\alpha=\left(\alpha_{1}, \alpha_{2}\right), \alpha_{j} \in \mathbb{Z}, \alpha \neq(0,0)\right\} .
$$

The inverse inertia tensor $\mathbf{J}$ maps $\sin _{\theta}^{*} \rightarrow \sin _{\theta}$, and depends on four parameters: $\theta, \tau \in$ $\mathbb{C}$, $(\Im m \tau>0)$ and $\epsilon=\left(\epsilon_{1}, \epsilon_{2}\right)$, where $0<\theta \epsilon_{a} \leq 1$ and $\epsilon_{a} \theta$ are irrational numbers. The components of $\mathbf{J}$ are given by the elliptic constants $J_{\alpha}=\wp_{\theta, \epsilon}(\alpha)$ (C.18)

$$
\mathbf{J}(\mathbf{S})=\sum_{\alpha \in \tilde{\mathbb{Z}}^{(2)}} \wp_{\theta, \epsilon}(\alpha) S_{-\alpha} T_{\alpha}
$$

The Hamiltonian has the form

$$
H=2 \pi^{2} \theta^{2} \int_{\mathcal{A}_{\theta}} \mathbf{S} \cdot \mathbf{J}(\mathbf{S})=-\frac{1}{2} \sum_{\alpha \in \tilde{\mathbb{Z}}^{(2)}} \wp_{\theta, \epsilon}(\alpha) S_{\alpha} S_{-\alpha} .
$$

Introduce a complex structure on the $\mathrm{NCT} \mathcal{A}_{\theta}$ depending on $\epsilon=\left(\epsilon_{1}, \epsilon_{2}\right)$ and $\tau$ such that for $X=\sum_{a} c_{a} T_{a}$ one has:

$$
\bar{\partial}_{\epsilon, \tau} X=\sum_{a}\left(\epsilon_{1} a_{1}+\epsilon_{2} a_{2} \tau\right) c_{a} T_{a}
$$


Then, in the Moyal representation, the operator $\mathbf{J}$ can be identified with the pseudo-differential operator

$$
\mathbf{J}(\mathbf{S})(x)=\wp\left(\theta \bar{\partial}_{\epsilon, \tau}\right) \mathbf{S}(x),
$$

and

$$
H=2 \pi^{2} \theta^{2} \int_{\mathcal{A}_{\theta}} \mathbf{S} \cdot \wp\left(\theta \bar{\partial}_{\epsilon, \tau}\right) \mathbf{S} .
$$

The equation of motion in the form of the Moyal brackets has the standard form

$$
\partial_{t} \mathbf{S}=\left\{\mathbf{S},\left(\wp\left(\theta \bar{\partial}_{\epsilon, \tau}\right) \mathbf{S}\right)\right\}^{\theta}:=\operatorname{ad}_{\mathbf{J}(\mathbf{S})}^{*} \mathbf{S},
$$

or, in the components,

$$
\partial_{t} S_{\alpha}=\sum_{\gamma \in \tilde{\mathbb{Z}}^{(2)}} S_{\gamma \wp_{\theta, \epsilon}(\gamma) S_{\alpha-\gamma}}
$$

2. The r-matrix and the Lax equation

Define formally the Lax operator

$$
L^{r o t}(z)=-\sum_{\alpha \in \tilde{\mathbb{Z}}^{(2)}} S_{\alpha} \varphi_{\theta, \epsilon}(\alpha, z) T_{\alpha}
$$

where $\varphi_{\theta, \epsilon}$ is given by (C.19). Below we formulate the conditions on the phase space, which show that $L^{r o t}(z)$ is well defined for $z \in E_{\tau}, z \neq 0$. Note that

$$
\int_{\mathcal{A}_{\theta}} L^{r o t}(z)=0
$$

The following Proposition is an infinite-dimensional analog of Proposition 3.1.

Proposition 4.1 The equations of motion (4.7) have the Lax form

$$
\partial_{t} L^{r o t}=\left[L^{r o t}, M^{r o t}\right]:=\left\{L^{r o t}, M^{r o t}\right\}^{\theta}
$$

with

$$
M^{r o t}(z)=-\sum_{\alpha \in \tilde{\mathbb{Z}}^{(2)}} S_{\alpha} f_{\theta, \epsilon}(\alpha, z) T_{\alpha}
$$

and $f_{\theta, \epsilon}($ C.21).

The proof is analogous to the finite-dimensional case. It is based on the Calogero functional equation (A.22).

Introduce the classical $r$-matrix on $\sin _{\theta} \otimes \sin _{\theta}$ as the following sum:

$$
r_{\theta, \epsilon, \tau}(z-w)=\sum_{\gamma \in \tilde{\mathbb{Z}}^{(2)}} \varphi_{\theta, \epsilon}(\gamma, z-w) T_{\gamma} \otimes T_{-\gamma},
$$

Lemma 3.1 and Proposition 3.2 have the following analogs for the NCT:

Proposition 4.2 a) The r-matrix (4.11) satisfies the classical Yang-Baxter equation (3.9).

b) The r-matrix defines the Poisson brackets for the entries of the Lax operator (4.8)

$$
\left\{L_{1}^{r o t}(z), L_{2}^{r o t}(w)\right\}_{1}=\left[r_{\theta, \epsilon, \tau}(z-w), L^{r o t}(z) \otimes I d+I d \otimes L^{r o t}(w)\right] .
$$

They are equivalent to the linear brackets (4.1) on $\sin _{\theta}^{*}$. 
The proof of Proposition 4.2(a,b) is similar to those of Lemma 3.1 and Proposition 3.2 and is based on the Fay identity (A.21).

3. The hierarchy of the Lax equations

In order to construct integrals of motion we first discuss the properties of the Lax operator. This operator is the meromorphic quasi-periodic function on $E_{\tau}$ with a simple pole

$$
\begin{gathered}
\left.\operatorname{Res} L^{r o t}(z)\right|_{z=0}=\mathbf{S}, \\
L^{r o t}(z+1)=U_{1}^{\epsilon_{1}} L^{r o t}(z) U_{1}^{-\epsilon_{1}}, \quad L^{r o t}(z+\tau)=U_{2}^{\epsilon_{2}} L^{r o t}(z) U_{2}^{-\epsilon_{2}} .
\end{gathered}
$$

where $U_{1}, U_{2}$ are the generators of $\mathrm{NCT} \mathcal{A}_{\theta}$ (C.1). (This follows from (4.8), (A.9) and (B.15).) It means that the integrals

$$
\int_{\mathcal{A}_{\theta}}\left(L^{r o t}(z)\right)^{j}
$$

are doubly periodic functions on $E_{\tau}$ with poles up to order $j$. Thereby, we have the expansion

$$
\int_{\mathcal{A}_{\theta}}\left(-L^{r o t}(z)\right)^{j}=I_{0, j}+\sum_{r=2}^{j} I_{r, j} \wp^{(r-2)}(z) .
$$

In particular,

$$
\int_{\mathcal{A}_{\theta}}\left(L_{\theta}\right)^{2}(z)=I_{0,2}+\wp(z) \int_{\mathcal{A}_{\theta}} \mathbf{S}^{2}, \text { where } I_{0,2}=2\left(\frac{i}{2 \pi \theta}\right)^{2} H
$$

Note that

$$
I_{j, j}=\int_{\mathcal{A}_{\theta}} \mathbf{S}^{j}
$$

are the Casimirs of the linear brackets.

The phase space $\mathcal{R}$ of the elliptic rotator is determined by the following properties of the integrals $I_{s, j}$

$$
\left.\mathcal{R}=\left\{\mathbf{S} \in \mathcal{A}_{\theta}^{*} \mid i\right) I_{s, j}<\infty, \text { ii) } \lim _{j \rightarrow \infty} \frac{I_{s, j+1}(\mathbf{S})}{I_{s, j}(\mathbf{S})}<1 \text { for all } s \leq j\right\} .
$$

The first condition means that the traces (4.13) of the Lax operator are well defined on $E_{\tau}$ for $z \neq 0$. In particular, we have

$$
I_{0,2}<\infty \text {, i.e. } \sum \wp_{\theta}(\alpha) S_{\alpha} S_{-\alpha}<\infty .
$$

We will use the second condition below to define the Casimir functions for the family of quadratic Poisson brackets.

Further, due to Lemma 3.1, an analog of Proposition 3.2 is the following.

Proposition 4.3 The quantities $I_{s, k}$ are pairwise Poisson commute.

The conservation laws $I_{s, k}$ generate commuting flows on the phase space $\mathcal{R}$ with respect to the linear brackets

$$
\partial_{s, k} \mathbf{S}=\left\{I_{s, k}, \mathbf{S}\right\}_{1}^{\theta}
$$

where $\partial_{s, k}$ stands for the corresponding time derivative $\partial_{t_{s, k}}$. We call these equations $E R_{\theta}$ hierarchy on the NCT.

One can show that all flows can be represented in the Lax form with $L^{\text {rot }}(4.8)$ and the corresponding $M_{s, k}$. Furthermore, there exists a set of the integrals of $J_{s, k}$-type. They are related to the integrals $I_{m, j}$ by the same formulae (3.15), (3.16). 


\subsection{Quadratic Poisson algebras and the bihamiltonian structure on NCT}

To define the quadratic Poisson algebra on the phase space $\mathcal{R}$ (4.14) we modify the Lax operator (4.8):

$$
L=-S_{0} \cdot T_{0}+L^{r o t}=S_{0} \cdot T_{0}+\sum_{\alpha} S_{-\alpha} \varphi_{\theta, \epsilon}(\alpha, z) T_{\alpha},
$$

where $S_{0}$ commutes with $S_{\alpha}$ with respect to the linear brackets. Due to the YB equation, the $r$-matrix (4.11) defines the quadratic Poisson brackets

$$
\left\{L_{1}(z), L_{2}(w)\right\}_{2}^{\theta}=\left[r_{\theta, \epsilon, \tau}(z-w), L_{1}(z) \otimes L_{2}(w)\right] .
$$

Again, we can get rid of the dependence on the spectral parameter.

Proposition 4.4 The quadratic Poisson brackets on the phase space $\mathcal{R}$ are defined as follows

$$
\begin{gathered}
\left\{S_{\alpha}, S_{0}\right\}_{2}^{\theta}=\sum_{\gamma \neq \alpha} S_{\alpha-\gamma} S_{\gamma}\left(\wp_{\theta, \epsilon}(\gamma)-\wp_{\theta, \epsilon}(\alpha-\gamma)\right) \mathbf{C}_{\theta}(\alpha, \gamma), \\
\left\{S_{\alpha}, S_{\beta}\right\}_{2}^{\theta}=S_{0} S_{\alpha+\beta} \mathbf{C}_{\theta}(\alpha, \beta)+\sum_{\gamma \neq \alpha,-\beta} S_{\alpha-\gamma} S_{\beta+\gamma} \mathbf{f}_{\theta, \epsilon}(\alpha, \beta, \gamma) \mathbf{C}_{\theta}(\gamma, \alpha-\beta),
\end{gathered}
$$

where $\mathbf{C}_{\theta}(\alpha, \gamma)$ is a structure constant of the $\sin _{\theta}$-algebra,

$$
\begin{gathered}
\mathbf{f}_{\theta, \epsilon}(\alpha, \beta, \gamma)=\zeta_{\theta, \epsilon}(\gamma)+\zeta_{\theta, \epsilon}(\beta-\alpha+\gamma)-\zeta_{\theta, \epsilon}(\beta+\gamma)+\zeta_{\theta, \epsilon}(\alpha-\gamma) \\
=-\frac{\vartheta^{\prime}(0) \vartheta_{\theta, \epsilon}(\alpha) \vartheta_{\theta, \epsilon}(\beta) \vartheta_{\theta, \epsilon}(\beta-\alpha+2 \gamma)}{\vartheta_{\theta, \epsilon}(\alpha-\gamma) \vartheta_{\theta, \epsilon}(\beta+\gamma) \vartheta_{\theta, \epsilon}(\beta-\alpha+\gamma) \vartheta_{\theta, \epsilon}(\gamma)},
\end{gathered}
$$

and $\vartheta_{\theta, \epsilon}, \zeta_{\theta, \epsilon}$ are the constants (C.17), (C.18).

Proof

The equality (4.18), being reduced to the coefficients in the front of $T_{a} \otimes T_{b}$, can be rewritten in the form (3.22) where the functions $\varphi_{\theta}(a, z)$ are replaced on $\varphi_{\theta, \epsilon}(a, z)$. Then using the cubic functional equations (A.27) and (A.28) we obtain to the algebra (4.19), (4.20). Due the definition of the phase space (4.15) the series in the right hand sides of (4.19), (4.20) converge.

We denote this Poisson algebra by $\mathcal{P}_{\theta, \epsilon, \tau}$. Set now $\epsilon_{1}=\epsilon_{2}=1$. The corresponding algebra $\mathcal{P}_{\theta, 1, \tau}$ can be considered as a special large $N$ limit of the finite-dimensional algebras $P_{N, \theta, \tau}(3.19)$, (3.20). For this we just replace the rational number $\theta=k / N$ by an arbitrary irrational number $0 \leq \theta<1$ in the algebra $P_{N, \theta, \tau}$.

Proposition 4.5 a) The linear (4.1) and quadratic (4.19), (4.20) Poisson brackets defined on $\mathcal{R}$ are compatible.

b) The ER $R_{\theta}$ hierarchy admits the bihamiltonian structure.

Proof.

One can shift $S_{0}+\lambda$ and define the family Poisson algebras $P_{\theta, \epsilon, \tau, \lambda}$. To define the Casimir elements on $P_{N, \theta, \tau, \lambda}$ we consider

$$
\log \operatorname{det}\left(S_{0}+\lambda+L^{r o t}(z)\right)=\log \left(S_{0}+\lambda\right)+\sum_{k=2}^{\infty} \frac{(-1)^{k+1}}{k\left(S_{0}+\lambda\right)^{k}} \operatorname{tr} L(z)^{k} .
$$


Then we come to the infinite set of Casimirs ${ }^{1}$

$$
\begin{gathered}
\tilde{C}_{0}^{(2)}\left(S_{0}+\lambda\right)=\log \left(S_{0}+\lambda\right)+\sum_{k=2}^{\infty} \frac{(-1)^{k+1}}{k\left(S_{0}+\lambda\right)^{k}} I_{0, k}, \\
\tilde{C}_{j}^{(2)}\left(S_{0}+\lambda\right)=\sum_{k=2}^{\infty} \frac{(-1)^{k+1}}{k\left(S_{0}+\lambda\right)^{k}} I_{j, k},(j=2,3, \ldots) .
\end{gathered}
$$

These functionals are well-defined on the phase space (4.14). This allows us to introduce the new set of conserved quantities

$$
\begin{aligned}
& \tilde{C}_{0}^{(2)}\left(S_{0}+\lambda\right)=\sum_{s} \tilde{h}_{s, 0} \lambda^{s}, \\
& \tilde{C}_{j}^{(2)}\left(S_{0}+\lambda\right)=\sum_{s} \tilde{h}_{s, j} \lambda^{s} .
\end{aligned}
$$

The latter Poisson commute with respect to the both types of brackets and give rise to the bihamiltonian structure of the hierarchy

$$
\left\{\tilde{h}_{s, j}, \mathbf{S}\right\}_{1}^{\theta}=-\left\{\tilde{h}_{s+1, j}, \mathbf{S}\right\}_{2}^{\theta} .
$$

In particular, we can represent the flow (4.6) in the form

$$
\partial_{t} \mathbf{S}=\left\{S_{0}, \mathbf{S}\right\}_{2}^{\theta}
$$

(see $(3.30))$.

This proposition concludes the proof of Theorem 2.1 for the hierarchy $E R_{\theta}$.

\section{$5 \quad$ Elliptic Rotators on $\operatorname{SDiff}\left(T^{2}\right)$}

\subsection{Description of the hierarchy}

In the "dispersionless" limit $\theta \rightarrow 0$ the Lie algebra $\sin _{\theta}$ turns to the Lie algebra $\operatorname{Ham}\left(T^{2}\right)$ of Hamiltonians on a two-dimensional torus, see (C.22). This algebra of Hamiltonian functions can be represented by the Lie algebra of the corresponding divergence-free vector fields $S V e c t\left(T^{2}\right)$. More precisely, to pass from $\operatorname{Ham}\left(T^{2}\right)$ to $S V$ ect $\left(T^{2}\right)$ one has to discard the constant Hamiltonians, but add the "flux" vector fields $\partial / \partial x_{1}$ and $\partial / \partial x_{2}$ corresponding to multivalued Hamiltonian functions $x_{1}$ and $x_{2}$ on the torus.

We define the elliptic rotator system $E R$ on the Lie group $\operatorname{SDiff}\left(T^{2}\right)$ by considering the limit $\theta \rightarrow 0$ of the $E R_{\theta}$-system described above. Let $\theta \rightarrow 0, \epsilon_{1,2} \rightarrow \infty$, such that

$$
\lim _{\theta \rightarrow 0}\left(\theta \epsilon_{1,2}\right)=\epsilon_{1,2}^{\prime}<1, \quad \epsilon_{1,2}^{\prime} \text { are irrational . }
$$

In what follows we drop the superscript '.

Let $\mathbf{S}=\sum_{\alpha} S_{-\alpha} \mathbf{e}(\alpha \cdot x) \in \operatorname{Ham}^{*}\left(T^{2}\right)$, where $\mathbf{e}(\alpha \cdot x)$ is the Fourier basis (C.23) of $\operatorname{Ham}^{*}\left(T^{2}\right)$. In the Fourier basis, the linear Poisson bracket assumes the form

$$
\left\{S_{\alpha}, S_{\beta}\right\}_{1}=(\alpha \times \beta) S_{\alpha+\beta}, \alpha \times \beta=\alpha_{1} \beta_{2}-\alpha_{2} \beta_{1} .
$$

\footnotetext{
${ }^{1}$ Since we pass from det to log det the basis of the Casimir functions differs from (3.24)
} 
The inverse inertia operator $\mathbf{J}: \operatorname{Ham}^{*}\left(T^{2}\right) \rightarrow \operatorname{Ham}\left(T^{2}\right)$ becomes

$$
\mathbf{J}: S_{\alpha} \rightarrow \wp_{\epsilon}(\alpha) S_{\alpha}, \wp_{\epsilon}(\alpha)=\wp\left(\epsilon_{1} \alpha_{1}+\epsilon_{2} \alpha_{2} \tau ; \tau\right),
$$

where $\alpha \in \tilde{\mathbb{Z}}^{(2)}$ (B.5). The operator is well defined since $\epsilon_{j}$ are irrational. In other words, the operator $\mathbf{J}$ is the pseudo-differential operator

$$
\mathbf{J}: \mathbf{S}(x) \rightarrow \wp\left(\bar{\partial}_{\epsilon, \tau}\right) \mathbf{S}(x),
$$

where $\bar{\partial}_{\epsilon, \tau}$ is the operator of the complex structure on the elliptic curve, commutative torus $T^{2}$. In fact, the complex structure depends on the ratio $\tau \epsilon_{2} / \epsilon_{1}$.

The quadratic Hamiltonian of the system is

$$
H=-\frac{1}{2} \sum_{\gamma} S_{\gamma \wp_{\epsilon}}(\gamma) S_{-\gamma}=-\frac{1}{2} \int_{T^{2}} \mathbf{S}\left(\wp\left(\bar{\partial}_{\epsilon, \tau}\right) \mathbf{S}\right)
$$

and the corresponding equations of motion are

$$
\partial_{t} \mathbf{S}=\left\{\mathbf{S}, \wp\left(\bar{\partial}_{\epsilon, \tau}\right) \mathbf{S}\right\}_{1}
$$

This equation is an elliptic analog of the hydrodynamics equation on the torus $T^{2}$, regarded as an elliptic curve, see Remark below. We call it the elliptic hydrodynamics.

Define the Lax operator

$$
L^{r o t}(x ; z)=-\sum_{\alpha \in \tilde{\mathbb{Z}}^{(2)}} S_{\alpha} \varphi_{\epsilon}(\alpha, z) \mathbf{e}(\alpha \cdot x), \quad \varphi_{\epsilon}(\alpha, z)=\varphi\left(\epsilon_{1} \alpha_{1}+\tau \epsilon_{2} \alpha_{2}, z\right) .
$$

The conditions on the phase space formulated below, see (5.12), ensure that the operator $L^{r o t}(x, z)$ is well defined for $z \in E_{\tau}, z \neq 0$. Note that

$$
\int_{T^{2}} L_{\epsilon}^{r o t}(z)=0
$$

Proposition 5.1 The equations of motion (5.4) have the dispersionless Lax representation

$$
L^{r o t}=\left\{L^{r o t}, M^{r o t}\right\}_{1}
$$

with $L^{\text {rot }}$ given by (5.5) and

$$
M^{r o t}(x ; z)=-\sum_{\alpha \in \tilde{\mathbb{Z}}^{(2)}} S_{\alpha} f_{\epsilon}(\alpha, z) \mathbf{e}(\alpha \cdot x),
$$

where

$$
f_{\epsilon}(\alpha, z)=f\left(\epsilon_{1} \alpha_{1}+\tau \epsilon_{2} \alpha_{2}, z\right)=\left.\mathbf{e}\left(\epsilon_{2} \alpha_{2}\right) \partial_{u} \phi(u, z)\right|_{u=\epsilon_{1} \alpha_{1}+\tau \epsilon_{2} \alpha_{2}} .
$$

The proof of Proposition 5.1 is the same as Proposition 3.1.

Note that operators $L^{r o t}$ and $M^{r o t}$ satisfy the quasi-periodicity properties

$$
\begin{aligned}
L^{r o t}\left(x_{1}, x_{2} ; z\right) & =L^{r o t}\left(x_{1}+\epsilon_{2}, x_{2} ; z+1\right), \\
L^{r o t}\left(x_{1}, x_{2} ; z\right) & =L^{r o t}\left(x_{1}, x_{2}-\epsilon_{1} ; z+\tau\right), \\
M^{r o t}\left(x_{1}, x_{2} ; z\right) & =M^{r o t}\left(x_{1}+\epsilon_{2}, x_{2} ; z+1\right),
\end{aligned}
$$


and

$$
M^{r o t}\left(x_{1}, x_{2} ; z\right)-M^{r o t}\left(x_{1}, x_{2}-\epsilon_{1} ; z+\tau\right)=2 \pi i L^{r o t}\left(x_{1}, x_{2}, z\right) .
$$

Furthermore, it follows from (5.7) and (5.8) that there exists the expansion

$$
\int_{T^{2}}\left(L^{r o t}(x, z)\right)^{k}=I_{0, k}+\sum_{s=2}^{k} I_{s, k} \wp^{(s-2)}(z) .
$$

The phase space $\mathcal{R}^{0}$ of the elliptic rotator on $\operatorname{SDiff}\left(T^{2}\right)$ can be described similarly to the phase space $\mathcal{R}$ for $S I N_{\theta}$-group, see (4.14):

$$
\left.\left.\mathcal{R}^{0}=\left\{\mathbf{S} \in \operatorname{Ham}^{*}\left(T^{2}\right) \mid i\right) I_{s, j}<\infty, \quad i i\right) \lim _{j \rightarrow \infty} \frac{I_{s, j+1}(\mathbf{S})}{I_{s, j}(\mathbf{S})}<1 \text { for all } s \leq j\right\} .
$$

Define the $r$-matrix on $T^{2} \otimes T^{2}$ by

$$
r_{\epsilon, \tau}(x, y ; z)=\sum_{\alpha \in \tilde{\mathbb{Z}}^{(2)}} \varphi_{\epsilon}(\alpha, z) \mathbf{e}(\alpha \cdot x) \mathbf{e}(-\alpha \cdot y), z \in E_{\tau} .
$$

Proposition 5.2 a) The r-matrix (5.13) satisfies the "dispersionless" YB equation

$$
\begin{gathered}
\left\{r_{\epsilon, \tau}(x, y ; z-w), r_{\epsilon, \tau}(x, v ; z)\right\}+\left\{r_{\epsilon, \tau}(x, y ; z-w), r_{\epsilon, \tau}(y, v ; w)\right\} \\
+\left\{r_{\epsilon, \tau}(x, v ; z), r_{\epsilon, \tau}(y, v ; w)\right\}=0 .
\end{gathered}
$$

b) In terms of the Lax operator (5.5) the canonical brackets on $T^{2}$ take the form

$$
\left\{L^{r o t}(x ; z), L^{r o t}(y ; w)\right\}=\left\{r_{\epsilon, \tau}(x, y, z-w), L^{r o t}(x ; z)\right\}+\left\{r(x, y, z-w) L^{r o t}(y ; w)\right\} .
$$

Proof

These statements are consequences of the Fay identity (A.21), cf. Proposition 4.2.

The form of the brackets (5.15) implies that

$$
\left\{\int_{T^{2}}\left(L^{r o t}(x, z)\right)^{k}, \int_{T^{2}}\left(L^{r o t}(x, z)\right)^{j}\right\}=0 .
$$

In turn, then (5.11) produces the infinite sequence of conservation laws $I_{s, k}$ in involution. They define the hierarchy of commuting flows

$$
\partial_{s, k} \mathbf{S}=\left\{I_{s, k}, \mathbf{S}\right\}_{1}
$$

which is the dispersionless limit of the hierarchy (4.16). The equations can be represented in the form of the dispersionless Lax equations.

\subsection{Quadratic Poisson algebras, and the bihamiltonian structure on $S \operatorname{Dif} f\left(T^{2}\right)$}

To describe the quadratic Poisson brackets, we first pass from the Lie algebraic Lax operator $L^{\text {rot }}$ to the operator

$$
L_{\epsilon}=-S_{0}+L^{r o t}(x ; z) .
$$

Define the quadratic Poisson algebra by the formula

$$
\left\{L_{\epsilon}(x ; z), L_{\epsilon}(y ; w)\right\}=\left\{r_{\epsilon, \tau}(x, y ; z-w), L_{\epsilon}(x ; z) L_{\epsilon}(y ; w)\right\} .
$$


Proposition 5.3 In terms of the Fourier modes, the Poisson algebra (5.18) has the form

$$
\begin{gathered}
\left\{S_{\alpha}, S_{0}\right\}_{2}=\sum_{\gamma \neq \alpha} S_{\alpha-\gamma} S_{\gamma}\left(\wp_{\epsilon}(\gamma)-\wp_{\epsilon}(\alpha-\gamma)\right)(\alpha \times \gamma), \\
\left\{S_{\alpha}, S_{\beta}\right\}_{2}=S_{0} S_{\alpha+\beta}(\alpha \times \beta)+\sum_{\gamma \neq \alpha,-\beta} S_{\alpha-\gamma} S_{\beta+\gamma} \mathbf{f}_{\epsilon}(\alpha, \beta, \gamma)(\gamma \times(\alpha-\beta)),
\end{gathered}
$$

where

$$
\begin{gathered}
\mathbf{f}_{\epsilon}(\alpha, \beta, \gamma)=\zeta_{\epsilon}(\gamma)+\zeta_{\epsilon}(\beta-\alpha+\gamma)-\zeta_{\epsilon}(\beta+\gamma)+\zeta_{\epsilon}(\alpha-\gamma) \\
=-\frac{\vartheta^{\prime}(0) \vartheta_{\epsilon}(\alpha) \vartheta_{\epsilon}(\beta) \vartheta_{\epsilon}(\beta-\alpha+2 \gamma)}{\vartheta_{\epsilon}(\alpha-\gamma) \vartheta_{\epsilon}(\beta+\gamma) \vartheta_{\epsilon}(\beta-\alpha+\gamma) \vartheta_{\epsilon}(\gamma)}, \\
\zeta_{\epsilon}(\gamma)=\zeta\left(\epsilon_{1} \gamma_{1}+\tau \epsilon_{2} \gamma_{2}\right), \vartheta_{\epsilon}(\gamma)=\zeta\left(\epsilon_{1} \gamma_{1}+\tau \epsilon_{2} \gamma_{2}\right) .
\end{gathered}
$$

The proof of this Proposition is similar to that of Proposition 3.2.

By the same trick as before we can show that the linear and the quadratic brackets are compatible. It allows us to construct the integral of the $\tilde{h}_{s, k}$ type and define the recurrence representation (4.22) for the bihamiltonian structure on the hierarchy. In particular, the equation (5.4) can be represented as

$$
\partial_{t} \mathbf{S}=\left\{S_{0}, \mathbf{S}\right\}_{2}
$$

\subsection{Rational limit of the elliptic hydrodynamics}

1. Description of the limit

So far we have been dealing with the elliptic curve $E_{\tau}$ parameterized by the two half-periods $\omega_{1}, \omega_{2}, \tau=\omega_{2} / \omega_{1}$. WE replace our main functions in the following way:

$$
\zeta_{\epsilon}(\gamma)=\zeta\left(\epsilon_{1} \gamma_{1} \omega_{1}+\epsilon_{2} \gamma_{2} \omega_{2} ; \omega_{1}, \omega_{2},\right), \wp_{\epsilon}(\gamma)=\wp\left(\epsilon_{1} \gamma_{1} \omega_{1}+\epsilon_{2} \gamma_{2} \omega_{2} ; \omega_{1}, \omega_{2},\right) .
$$

Now we are going to consider the rational limit of the elliptic curves, i.e., degeneration $\lim \omega_{1,2} \rightarrow$ $\infty$. We look at the double scaling limit

$$
\lim \epsilon_{1,2} \rightarrow 0, \lim \omega_{1,2} \rightarrow \infty, \lim \epsilon_{1} \omega_{1}=1, \lim \epsilon_{2} \omega_{2}=\tau .
$$

Then $\lim \omega_{1,2} \rightarrow \infty$ leads to the rational degeneration of the elliptic functions (5.21)

$$
\lim _{\omega_{1,2} \rightarrow \infty} \zeta_{\epsilon}(\gamma)=\frac{1}{\gamma_{\tau}}, \quad \lim _{\omega_{1,2} \rightarrow \infty} \wp_{\epsilon}(\gamma)=\frac{1}{\gamma_{\tau}^{2}}, \quad \gamma_{\tau}=\gamma_{1}+\gamma_{2} \tau
$$

This implies, in particular, that the inverse inertia tensor assumes the form

$$
\mathbf{J}(\mathbf{S})=\bar{\partial}^{-2} \mathbf{S}\left(x_{1}, x_{2}\right)=\sum_{\alpha \in \tilde{\mathbb{Z}}^{(2)}} \frac{1}{\alpha_{\tau}^{2}} S_{-\alpha} T_{\alpha},
$$

where $\alpha_{\tau}=\alpha_{1}+\alpha_{2} \tau$. The equations of motion are defined by the corresponding quadratic Hamiltonian

$$
H=-\frac{1}{2} \int_{T^{2}} \mathbf{S} \bar{\partial}^{-2} \mathbf{S}=-\frac{1}{2} \sum_{\alpha \in \tilde{\mathbb{Z}}^{(2)}} \frac{1}{\alpha_{\tau}^{2}} S_{\alpha} S_{-\alpha} .
$$

with respect to the linear Poisson bracket on $\operatorname{Ham}\left(T^{2}\right)$ :

$$
\left.\partial_{t} \mathbf{S}=\left\{\mathbf{S}, \bar{\partial}^{-2} \mathbf{S}\right)\right\}_{1}
$$


In the Fourier components the latter becomes

$$
\partial_{t} S_{\alpha}=\sum_{\gamma \in \tilde{\mathbb{Z}}^{(2)}} S_{\gamma} \frac{1}{\gamma_{\tau}^{2}} S_{\alpha-\gamma}
$$

We call these equations the modified hydrodynamics on the torus $T^{2}$.

Remark 5.1 Recall that the standard hydrodynamics of an ideal fluid on the torus $T^{2}$ is given by the Euler equation

$$
\partial_{t} \mathbf{S}=\left\{\mathbf{S}, \Delta^{-1} \mathbf{S}\right\}_{1},
$$

on the vorticity function $\mathbf{S}$. The definition of the Laplace operator uses metric, while to define the modified hydrodynamics (5.24) we need to fix a complex structure on $T^{2}$.

Now consider the Lax representation for the modified hydrodynamics. In the double scaling limit we have

$$
\begin{gathered}
\lim \varphi_{\epsilon}(\alpha, z)=\exp \left(2 \pi i \alpha_{1} z\right)\left(\frac{1}{\alpha_{\tau}}+\frac{1}{z}\right), \\
\lim f_{\epsilon}(\alpha, z)=-\exp \left(2 \pi i \alpha_{1} z\right) \frac{1}{\alpha_{\tau}^{2}}
\end{gathered}
$$

Therefore, the Lax pair (5.6) in the rational limit is

$$
\begin{gathered}
L\left(x_{1}, x_{2} ; z\right)=-\sum_{\alpha} S_{\alpha} \exp \left(2 \pi i \alpha_{1} z\right)\left(\frac{1}{\alpha_{\tau}}+\frac{1}{z}\right) T_{\alpha} . \\
M\left(x_{1}, x_{2}\right)=-\sum_{\alpha} S_{\alpha} \exp \left(2 \pi i \alpha_{1} z\right) \frac{1}{\alpha_{\tau}^{2}} T_{\alpha} .
\end{gathered}
$$

We can drop the exponents in the both operators and come to the following expressions

$$
L\left(x_{1}, x_{2} ; z\right)=-\bar{\partial}^{-1} \mathbf{S}\left(x_{1}, x_{2}\right)-\frac{1}{z} \mathbf{S}\left(x_{1}, x_{2}\right),
$$

and

$$
M\left(x_{1}, x_{2}\right)=-\bar{\partial}^{-2} \mathbf{S}\left(x_{1}, x_{2}\right) .
$$

It is easy to see that the dispersionless Lax equation

$$
\partial_{t} L\left(x_{1}, x_{2} ; z\right)=\left\{L\left(x_{1}, x_{2} ; z\right), M\left(x_{1}, x_{2}\right)\right\}
$$

is equivalent to the equations (5.24).

The infinite set of the integrals of motion comes from the decomposition

$$
(-1)^{k} \int_{T^{2}} L^{k}\left(x_{1}, x_{2} ; z\right) d x_{1} d x_{2}=I_{0, k}+\sum_{s=2}^{k} I_{m, k} z^{-m} .
$$

The corresponding infinite hierarchy of the modified hydrodynamics

$$
\partial_{s, t} \mathbf{S}=\left\{I_{s, k}, \mathbf{S}\right\}_{1}
$$

has the dispersionless Lax form representation. 


\section{Bihamiltonian structure}

It turns out that the bihamiltonian structure survives in this limit. In fact, we have the following quadratic Poisson algebra $\mathcal{P}_{0, \tau}$ on the commutative torus $T^{2}$

$$
\begin{gathered}
\left\{S_{\alpha}, S_{0}\right\}_{2}=\sum_{\gamma \neq \alpha} S_{\alpha-\gamma} S_{\gamma}\left(\frac{1}{\gamma_{\tau}^{2}}-\frac{1}{\left(\alpha_{\tau}-\gamma_{\tau}\right)^{2}}\right)(\alpha \times \gamma), \\
\left\{S_{\alpha}, S_{\beta}\right\}_{2}=S_{0} S_{\alpha+\beta}(\alpha \times \beta)+\sum_{\gamma \neq \alpha,-\beta} S_{\alpha-\gamma} S_{\beta+\gamma} f(\alpha, \beta, \gamma)(\gamma \times(\alpha-\beta)),
\end{gathered}
$$

where

$$
f(\alpha, \beta, \gamma)=\left(\frac{1}{\gamma_{\tau}}+\frac{1}{\beta_{\tau}-\alpha_{\tau}+\gamma_{\tau}}-\frac{1}{\beta_{\tau}+\gamma_{\tau}}+\frac{1}{\alpha_{\tau}-\gamma_{\tau}}\right), \quad \gamma_{\tau}=\gamma_{1}+\tau \gamma_{2} .
$$

Similarly to the above, we can consider a one-parametric family of the quadratic algebras by replacing $S_{0} \rightarrow S_{0}+\lambda$. Note that it degenerates to the standard Poisson brackets on $T^{2}$ in the limit $\lambda \rightarrow \infty$. This allows us to define the bihamiltonian structure for the hierarchy of the modified hydrodynamics. In particular, the equation (5.24) can be rewritten in the form as

$$
\partial_{t} \mathbf{S}=\left\{S_{0}, \mathbf{S}\right\}_{2} .
$$

Such a bihamiltonian structure is a curious feature in the modified hydrodynamics, emphasizing its drastic difference from the classical hydrodynamics.

\section{Quantum counterparts}

In this section we present two associative algebras "quantizing" the discussed above two Poisson algebras in the case of the NCT. It is easy with the linear bracket. Indeed, the quantization of the Lie-Poisson algebra on $\sin _{\theta}^{*}$ leads to the universal enveloping algebra $\sin _{\theta}$.

Replace the classical variables $\mathbf{S}$ on the $\mathrm{NCT} \mathcal{A}_{\theta}$ by non-commuting variables $\hat{\mathbf{S}}$. Now we consider the quantization of the quadratic Poisson algebra $P_{\theta, \epsilon, \tau}$ and construct the associative algebra $\hat{P}_{\theta, \epsilon, \tau, \hbar}$, where $\hbar \in E_{\tau}$ is the deformation parameter. For this we introduce the quantum $R$-matrix related to the group $S I N_{\theta}$. The quantum Yang-Baxter equation defined on $\operatorname{GL}(N, \mathbb{C})$ $[13,14]$ is generalized to $S I N_{\theta}$ in the following way. The quantum $R$-matrix on $S I N_{\theta} \otimes S I N_{\theta}$ assumes the form

$$
R(z, w)=\sum_{c} \varphi_{\theta, \epsilon}(c, z-w \mid \hbar) T_{c} \otimes T_{-c},
$$

where $T_{c}$ is the basis of $S I N_{\theta}$ and the main ingredient in our construction is the function

$$
\varphi_{\theta, \epsilon}(c, z \mid \hbar)=\mathbf{e}_{\theta}\left(\epsilon_{2} c_{2} \theta\right) \phi\left(\left(\epsilon_{1} c_{1}+\tau \epsilon_{2} c_{2}\right) \theta+\hbar, z\right), \quad \hbar \in E_{\tau} .
$$

Note that, in contrast with the classical $r$-matrix (4.11), there is an additional term $\varphi_{\theta, \epsilon}(0, z-w \mid \hbar) T_{0} \otimes T_{0}$.

Proposition 6.1 The matrix $R$ satisfies the quantum $Y B$ equation

$$
R_{12}(z-w) R_{13}(z) R_{23}(w)=R_{23}(w) R_{13}(z) R_{12}(z-w) .
$$


Proof.

Consider the coefficients in front of $T_{a} \otimes T_{b} \otimes T_{c}$ in the l.h.s of (6.1). It vanishes if $a+b+c \neq 0$. Therefore (6.1) implies

$$
\begin{gathered}
\mathbf{e}_{\theta}(a \times b+2 b \times c) \varphi_{\theta, \epsilon}(c, z-w \mid \hbar) \varphi_{\theta, \epsilon}(a-c, z \mid \hbar) \varphi_{\theta, \epsilon}(b+c, w \mid \hbar) \\
-\mathbf{e}_{\theta}(a \times b+2 b \times f) \varphi_{\theta, \epsilon}(f, z-w \mid \hbar) \varphi_{\theta, \epsilon}(a-f, z \mid \hbar) \varphi_{\theta, \epsilon}(b+f, w \mid \hbar)=0
\end{gathered}
$$

Here $a, b$ are fixed and $c, f$ are arbitrary elements of the lattice $\mathbb{Z}_{\theta, \epsilon}(\tau)$ (C.16). Choose $f$ in the form $f=a-b-c$ to obtain

$$
\begin{gathered}
\mathbf{e}_{\theta}(a \times b+2 b \times c)\left(\varphi_{\theta, \epsilon}(c, z-w \mid \hbar) \varphi_{\theta, \epsilon}(a-c, z \mid \hbar) \varphi_{\theta, \epsilon}(b+c, w \mid \hbar)\right. \\
\left.-\varphi_{\theta, \epsilon}(a-b-c, z-w \mid \hbar) \varphi_{\theta, \epsilon}(a-c, w \mid \hbar) \varphi_{\theta, \epsilon}(b+c, z \mid \hbar)\right)=0 .
\end{gathered}
$$

This equality can be transform to the form

$$
\begin{gathered}
\varphi_{\theta, \epsilon}(a+2 \hbar, z \mid \hbar) \varphi_{\theta, \epsilon}(b, w \mid \hbar) \mathbf{e}_{\theta}(a \times b+2 b \times c) \\
\times\left(\zeta_{\theta, \epsilon}(c+\hbar)-\zeta_{\theta, \epsilon}(a-b-c+\hbar)+\zeta_{\theta, \epsilon}(a-c+\hbar)-\zeta_{\theta, \epsilon}(b+c+\hbar)\right)=0
\end{gathered}
$$

by means of (A.27). If $b=0$ then the l.h.s. vanishes and we come to (6.1). Let $b \neq 0$ and consider the shift $c \rightarrow c+j b$, where $j \in \mathbb{Z}$. The shift does not change the exponential factor. Take the sum over the orbit generated by the shifts

$$
\sum_{j \in \mathbb{Z}} \zeta_{\theta, \epsilon}(c+j b+\hbar)-\zeta_{\theta, \epsilon}(a-b-j b-c+\hbar)+\zeta_{\theta, \epsilon}(a-c+j b+\hbar)-\zeta_{\theta, \epsilon}(b+c+j b+\hbar) .
$$

One can see that the neighboring terms in the series vanish and we come to (6.1) for an arbitrary $b$.

This Proposition allows us to define the associative algebra $\hat{P}_{\theta, \epsilon, \tau, \hbar}$ by the relation

$$
R(z-w) L_{1}^{\hbar}(z) L_{2}^{\hbar}(w)=L_{2}^{\hbar}(w) L_{1}^{\hbar}(z) R(z-w)
$$

where

$$
L^{\hbar}(z)=\hat{S}_{0} \varphi_{\theta \epsilon}(0, z \mid \hbar) T_{0}+\sum_{\alpha} \hat{S}_{-\alpha} \varphi_{\theta, \epsilon}(\alpha, z \mid \hbar) T_{\alpha}
$$

In order to obtain the relations in $\hat{P}_{\theta, \epsilon, \tau, \hbar}$ one should exclude the spectral parameters $z, w$ from (6.2).

Lemma 6.1 The relations in the associative algebra $\hat{P}_{\theta, \tau, \hbar}$ assume the form

$$
\sum_{c} \hat{S}_{a-c} \hat{S}_{b+c} \mathbf{e}_{\theta}(c \cdot(a-b)) \mathbf{f}_{\theta, \epsilon}(a, b, c \mid \hbar)=0, \text { for any } a, b \in \mathbb{Z} \oplus \mathbb{Z}
$$

where

$$
\begin{gathered}
\mathbf{f}_{\theta, \epsilon}(a, b, c \mid \hbar)=\zeta_{\theta, \epsilon}(c+\hbar)-\zeta_{\theta, \epsilon}(a-b-c+\hbar)-\zeta_{\theta, \epsilon}(b+c+\hbar)+\zeta_{\theta, \epsilon}(a-c+\hbar) \\
=-\frac{\vartheta^{\prime}(0) \vartheta_{\theta, \epsilon}(\alpha+2 \hbar) \vartheta_{\theta, \epsilon}(\beta) \vartheta_{\theta, \epsilon}(\beta-\alpha+2 \gamma)}{\vartheta_{\theta, \epsilon}(\alpha-\gamma+\hbar) \vartheta_{\theta, \epsilon}(\beta+\gamma+\hbar) \vartheta_{\theta, \epsilon}(\beta-\alpha+\gamma+\hbar) \vartheta_{\theta, \epsilon}(\gamma+\hbar)} .
\end{gathered}
$$

and $\vartheta_{\theta, \epsilon}, \zeta_{\theta, \epsilon}($.$) are the constants (C.17), (C.18).$ 
Proof.

Consider in (6.2) the matrix element $T_{a} \otimes T_{b}$. We come to the relation

$$
\begin{gathered}
\sum_{c} \hat{S}_{a-c} \hat{S}_{b+c}\left\{\varphi_{\theta, \epsilon}(c, z-w \mid \hbar) \varphi_{\theta, \epsilon}(a-c, z \mid \hbar) \varphi_{\theta, \epsilon}(b+c, w \mid \hbar)\right. \\
\left.-\varphi_{\theta, \epsilon}(a-b-c, z-w \mid \hbar) \varphi_{\theta, \epsilon}(a-c, w \mid \hbar) \varphi_{\theta}(b+c, z \mid \hbar)\right\} \mathbf{e}_{\theta}(c \cdot(a-b))=0 .
\end{gathered}
$$

The expression in the brackets \{\} is equal to

$$
\begin{gathered}
\mathbf{e}_{\theta}((a+2 \hbar) z+(b+\hbar) w)\left\{\phi_{\theta, \epsilon}(c, z-w \mid \hbar) \phi_{\theta, \epsilon}(a-c, z \mid \hbar) \phi_{\theta, \epsilon}(b+c, z-w \mid \hbar)\right. \\
\left.-\phi_{\theta, \epsilon}(a-b-c, z-w \mid \hbar) \phi_{\theta, \epsilon}(a-c, w \mid \hbar) \phi_{\theta, \epsilon}(b+c, z \mid \hbar)\right\}
\end{gathered}
$$

where $\phi_{\theta, \epsilon}(c, z \mid \hbar)=\phi\left(\left(\epsilon_{1} c_{1}+\tau \epsilon_{2} c_{2}\right) \theta+\hbar, z\right)$. Finally, by using (A.27) we come from (6.5) to (6.4).

We rewrite (6.3) in the form most resembling the original Sklyanin relations [14]. Let $\mathfrak{A}(\alpha, \beta)$ (respectively, $\mathfrak{S}(\alpha, \beta)$ ) be the operator of antisymmetrization (respectively, symmetrization) with respect to the permutations of two indices $(\alpha, \beta)$.

Proposition 6.2 The relations (6.4) are equivalent to the commutator relations

$$
\begin{gathered}
{\left[\hat{S}_{0}, \hat{S}_{b}\right]=-\sum_{\gamma \neq b} \hat{S}_{-\gamma} \hat{S}_{b+\gamma} \mathbf{e}_{\theta}(b \cdot \gamma) \frac{\mathbf{f}_{\theta, \epsilon}(0, b, \gamma \mid \hbar)}{\mathbf{f}_{\theta, \epsilon}(0, b, 0 \mid \hbar)}} \\
{\left[\hat{S}_{\alpha}, \hat{S}_{\beta}\right]=\mathfrak{A}(\alpha, \beta)\left\{\left(\hat{S}_{\alpha+\beta} \hat{S}_{0} \mathbf{e}_{\theta}(-\beta \cdot \alpha)-\hat{S}_{0} \hat{S}_{\alpha+\beta} \mathbf{e}_{\theta, \epsilon}(\beta \cdot \alpha)\right) \frac{\mathbf{f}_{\theta, \epsilon}(\alpha, \beta, \alpha \mid \hbar)}{\mathbf{f}_{\theta, \epsilon}(\alpha, \beta, 0 \mid \hbar)}\right.} \\
\left.+\sum_{\gamma \neq \alpha,-\beta}\left(\hat{S}_{\alpha-\gamma} \hat{S}_{\beta+\gamma} \mathbf{e}_{\theta}(\gamma \cdot(\alpha-\beta))-\hat{S}_{\beta+\gamma} \hat{S}_{\alpha-\gamma} \mathbf{e}_{\theta}(-\gamma \cdot(\alpha-\beta))\right) \frac{\mathbf{f}_{\theta, \epsilon}(\alpha, \beta, \gamma \mid \hbar)}{\mathbf{f}_{\theta, \epsilon}(\alpha, \beta, 0 \mid \hbar)}\right\},
\end{gathered}
$$

where $\mathbf{C}_{\theta}(\alpha, \beta)$ are $\operatorname{sl}(N, \mathbb{C})$ structure constants (B.8), and

$$
\begin{gathered}
\mathfrak{S}(\alpha, \beta)\left\{\left(\hat{S}_{\alpha+\beta} \hat{S}_{0} \mathbf{e}_{\theta}(-\beta \cdot \alpha)-\hat{S}_{0} \hat{S}_{\alpha+\beta} \mathbf{e}_{\theta, \epsilon}(\beta \cdot \alpha)\right) \frac{\mathbf{f}_{\theta, \epsilon}(\alpha, \beta, \alpha \mid \hbar)}{\mathbf{f}_{\theta, \epsilon}(\alpha, \beta, 0 \mid \hbar)}\right. \\
\left.+\sum_{\gamma \neq \alpha,-\beta}\left(\hat{S}_{\alpha-\gamma} \hat{S}_{\beta+\gamma} \mathbf{e}_{\theta}(\gamma \cdot(\alpha-\beta))-\hat{S}_{\beta+\gamma} \hat{S}_{\alpha-\gamma} \mathbf{e}_{\theta}(-\gamma \cdot(\alpha-\beta))\right) \frac{\mathbf{f}_{\theta, \epsilon}(\alpha, \beta, \gamma \mid \hbar)}{\mathbf{f}_{\theta, \epsilon}(\alpha, \beta, 0 \mid \hbar)}\right\}=0 .
\end{gathered}
$$

Proof.

The relation (6.3) can be rewritten in the form

$$
\sum_{c}\left(\hat{S}_{a-c} \hat{S}_{b+c} \mathbf{e}_{\theta}(c \cdot(a-b))-\hat{S}_{b+c} \hat{S}_{a-c} \mathbf{e}_{\theta}(c \cdot(a-b))\right) \mathbf{f}_{\theta, \epsilon}(a, b, c \mid \hbar)=0 .
$$

due to the equality $\mathbf{f}_{\theta, \epsilon}(a, b, c \mid \hbar)=-\mathbf{f}_{\theta, \epsilon}(a, b, a-b-c \mid \hbar)$. For the case $a=0$ this relation assumes the form (6.6). To come to (6.7) we single out the two terms with $c=0$ and put them in the left hand side. In the right hand side we first write down the terms with $c=a$ and $c=-b$ and obtain the required relations. 
It would be interesting to describe a quantum version of the whole bihamiltonian structure for elliptic rotators on $\sin ^{*}$ or $\operatorname{Ham}\left(T^{2}\right)$. Posing the problem more generally, the bihamiltonian recursion precedure of generating the conserved quantities from a Casimir function for a linear family of Poisson structures (see [19]) might have a quantum analog as an expansion of a central element for a linear family of associative algebras. The latter seems to be a very strong requirement on a pair of associative algebras and it would be very interesting to find any non-trivial example of this kind. Namely, one is looking for a pair of associative algebras, such that their mixed associator satisfies some consistency condition. This would provide the most straightforward quantization for a system with a bihamiltonian structure.

\section{Acknowledgments}

We are grateful for hospitality to the Max-Planck-Institut fur Mathematik in Bonn, where most of this work was done. B.K. is also grateful for hospitality to the University of Nice and IHES in Bures-sur-Yvette. The work of B.K. was partially supported by an NSERC research grant. The work of A.L. and M.O. was supported in part by grants RFBR-03-02-17554, NSch-1999-2003.2 and CRDF 12729.

\section{Appendix}

\subsection{Appendix A. Elliptic functions.}

Here we summarize the main formulae for elliptic functions. Consider an elliptic curve

$$
E_{\tau}=\mathbb{C} /(\mathbb{Z}+\tau \mathbb{Z}), \quad q=\mathbf{e}(\tau)=\exp (2 \pi i \tau) .
$$

The basic element in our consideration is the theta function:

$$
\begin{gathered}
\vartheta(z ; \tau)=q^{\frac{1}{8}} \sum_{n \in \mathbf{Z}}(-1)^{n} e^{\pi i(n(n+1) \tau+2 n z)}= \\
q^{\frac{1}{8}} e^{-\frac{i \pi}{4}}\left(e^{i \pi z}-e^{-i \pi z}\right) \prod_{n=1}^{\infty}\left(1-q^{n}\right)\left(1-q^{n} e^{2 i \pi z}\right)\left(1-q^{n} e^{-2 i \pi z}\right) .
\end{gathered}
$$

1. The Weierstrass functions:

$$
\sigma(z ; \tau)=\exp \left(\eta z^{2}\right) \frac{\vartheta(z ; \tau)}{\vartheta^{\prime}(0 ; \tau)}
$$

where

$$
\begin{gathered}
\eta(\tau)=-\frac{1}{6} \frac{\vartheta^{\prime \prime \prime}(0 ; \tau)}{\vartheta^{\prime}(0 ; \tau)} . \\
\zeta(z ; \tau)=\partial_{z} \log \vartheta(z ; \tau)+2 \eta(\tau) z, \quad \zeta(z ; \tau) \sim \frac{1}{z}+O\left(z^{3}\right) . \\
\wp(z ; \tau)=-\partial_{z} \zeta(z ; \tau) . \\
\wp(u ; \tau)=\frac{1}{u^{2}}++\sum_{j, k}^{\prime}\left(\frac{1}{(j+k \tau+u)^{2}}-\frac{1}{(j+k \tau)^{2}}\right) .
\end{gathered}
$$


2. Function $\phi$ :

$$
\phi(u, z)=\frac{\vartheta(u+z) \vartheta^{\prime}(0)}{\vartheta(u) \vartheta(z)}
$$

It has a pole at $z=0$ and the expansion

$$
\phi(u, z)=\frac{1}{z}+\zeta(u \mid \tau)+2 \eta(\tau) z+\frac{z}{2}\left((\zeta(u ; \tau)+2 \eta(\tau) z)^{2}-\wp(u)\right)+\ldots,
$$

as $z \rightarrow 0$. Furthermore,

$$
\begin{gathered}
\phi(u, z)^{-1} \partial_{u} \phi(u, z)=\zeta(u+z)-\zeta(u)+2 \eta(\tau) z . \\
\phi(u, z)=\exp (-2 \eta u z) \frac{\sigma(u+z)}{\sigma(u) \sigma(z)} \\
\phi(u, z) \phi(-u, z)=\wp(z)-\wp(u) .
\end{gathered}
$$

3. Quasi-periodicity:

$$
\begin{gathered}
\vartheta(z+1 ; \tau)=-\vartheta(z ; \tau), \quad \vartheta(z+\tau ; \tau)=-\mathbf{e}\left(-\frac{1}{2} \tau-z\right) \vartheta(z ; \tau) . \\
\zeta(z+1 ; \tau)=\zeta(z ; \tau)-2 \eta, \quad \zeta(z+\tau ; \tau)=\zeta(z ; \tau)-2(\pi i+\eta \tau) . \\
\wp(u+1 ; \tau)=\wp(u+\tau ; \tau)=\wp(u ; \tau) . \\
\phi(u+1, z)=\phi(u, z), \quad \phi(u+\tau, z)=\mathbf{e}(-z) \phi(u, z) .
\end{gathered}
$$

4. Parity:

$$
\begin{gathered}
\vartheta(-z ; \tau)=-\vartheta(z ; \tau) . \\
\zeta(-z ; \tau)=-\zeta(z ; \tau) . \\
\wp(-u ; \tau)=\wp(u ; \tau) . \\
\phi(-u,-z)=-\phi(u, z) .
\end{gathered}
$$

5. The Fay three-section formula:

$$
\phi\left(u_{1}, z_{1}\right) \phi\left(u_{2}, z_{2}\right)-\phi\left(u_{1}+u_{2}, z_{1}\right) \phi\left(u_{2}, z_{2}-z_{1}\right)-\phi\left(u_{1}+u_{2}, z_{2}\right) \phi\left(u_{1}, z_{1}-z_{2}\right)=0 .
$$

Particular cases of this formula are (A.12), the Calogero functional equation

$$
\phi(u, z) \partial_{v} \phi(v, z)-\phi(v, z) \partial_{u} \phi(u, z)=(\wp(v)-\wp(u)) \phi(u+v, z),
$$

and

$$
\phi\left(u_{1}, z\right) \phi\left(u_{2}, z\right)-\phi\left(u_{1}+u_{2}, z\right)\left(\zeta\left(u_{1}\right)+\zeta\left(u_{2}\right)-2 \eta(\tau)\left(u_{1}+u_{2}\right)\right)+\partial_{z} \phi\left(u_{1}+u_{2}, z\right)=0 .
$$

For $u_{1}+u_{2}+u_{3}=0$,

$$
\phi\left(u_{1}, z\right) \phi\left(u_{2}, z\right) \phi\left(u_{3}, z\right)=\left[\wp(z)-\wp\left(u_{3}\right)\right]\left[\zeta\left(u_{1}\right)+\zeta\left(u_{2}\right)+\zeta\left(u_{3}-z\right)+\zeta(z)\right],
$$


as follows from (A.10), (A.12), and (A.23). Then

$$
\begin{gathered}
\left.\phi\left(u_{1}, z\right) \phi\left(u_{2}, z\right) \phi\left(u_{3}, z\right)\right|_{z \rightarrow 0}=\frac{1}{z^{3}}+\frac{1}{z^{2}}\left[\zeta\left(u_{1}\right)+\zeta\left(u_{2}\right)+\zeta\left(u_{3}\right)\right] \\
-\frac{1}{2} \wp^{\prime}\left(u_{3}\right)-\wp\left(u_{3}\right)\left[\zeta\left(u_{1}\right)+\zeta\left(u_{2}\right)+\zeta\left(u_{3}\right)\right]+O(z) .
\end{gathered}
$$

¿From (A.21) and (A.10) we have

$$
\phi\left(u_{1}, z\right) \phi\left(u-u_{1}, z\right)=\phi(u, z)\left(\zeta\left(u_{1}\right)+\zeta\left(u-u_{1}\right)-\zeta(u+z)+\zeta(z)\right) .
$$

Another important relation is

$$
\begin{gathered}
\phi(v, z-w) \phi\left(u_{1}-v, z\right) \phi\left(u_{2}+v, w\right)-\phi\left(u_{1}-u_{2}-v, z-w\right) \phi\left(u_{2}+v, z\right) \phi\left(u_{1}-v, w\right)= \\
\phi\left(u_{1}, z\right) \phi\left(u_{2}, w\right) f\left(u_{1}, u_{2}, v\right),
\end{gathered}
$$

where

$$
f\left(u_{1}, u_{2}, v\right)=\zeta(v)-\zeta\left(u_{1}-u_{2}-v\right)+\zeta\left(u_{1}-v\right)-\zeta\left(u_{2}+v\right) .
$$

One can rewrite the last function as

$$
f\left(u_{1}, u_{2}, v\right)=-\frac{\vartheta^{\prime}(0) \vartheta\left(u_{1}\right) \vartheta\left(u_{2}\right) \vartheta\left(u_{2}-u_{1}+2 v\right)}{\vartheta\left(u_{1}-v\right) \vartheta\left(u_{2}+v\right) \vartheta\left(u_{2}-u_{1}+v\right) \vartheta(v)} .
$$

To prove (A.27) one should consider the expression

$$
\frac{\phi(v, z-w) \phi\left(u_{1}-v, z\right) \phi\left(u_{2}+v, w\right)-\phi\left(u_{1}-u_{2}-v, z-w\right) \phi\left(u_{2}+v, z\right) \phi\left(u_{1}-v, w\right)}{\phi\left(u_{1}, z\right) \phi\left(u_{2}, w\right)} .
$$

It is a doubly periodic function in $z$ and $w$ without poles. Therefore, it is a constant, which depends on $u_{1}, u_{2}, v$. This constant is equal to $f\left(u_{1}, u_{2}, v\right)$ (A.28). It is easy to check that the elliptic functions (A.28) and (A.29) coincide.

A particular case of (A.27), which corresponds to the case $v=u_{1}$ (or $v=-u_{2}$ ), is the Fay identity (A.21). Another particular case comes from $u_{2}=0$ (or $\left.u_{1}=0\right)$ :

$$
\begin{gathered}
\phi(v, z-w) \phi(u-v, z) \phi(v, w)-\phi(u-v, z-w) \phi(v, z) \phi(u-v, w)= \\
\phi\left(u_{1}, z\right)(\wp(v)-\wp(u-v)) .
\end{gathered}
$$

\subsection{Appendix B. Elliptic constants related to $\mathrm{gl}(N, \mathbb{C})$.}

Let $1 \leq k<N$ be a coprime number with respect to $N$ and $\operatorname{set} \theta=\frac{k}{N}$. Define

$$
\begin{gathered}
\mathbf{e}_{\theta}(z)=\exp (2 \pi i \theta z) \\
Q=\operatorname{diag}\left(\mathbf{e}_{\theta}(1), \ldots, \mathbf{e}_{\theta}(m), \ldots, 1\right) \\
\Lambda=\left(\begin{array}{ccccc}
0 & 1 & 0 & \cdots & 0 \\
0 & 0 & 1 & \cdots & 0 \\
\vdots & \vdots & \ddots & \ddots & \vdots \\
0 & 0 & 0 & \cdots & 1 \\
1 & 0 & 0 & \cdots & 0
\end{array}\right) .
\end{gathered}
$$


Consider a finite two-dimensional lattice of order $N^{2}$

$$
\mathbb{Z}_{N}^{(2)}=\mathbb{Z} / N \mathbb{Z} \oplus \mathbb{Z} / N \mathbb{Z}
$$

The matrices

$$
T_{a}=\frac{1}{2 \pi i \theta} \mathbf{e}_{\theta}\left(\frac{a_{1} a_{2}}{2}\right) Q^{a_{1}} \Lambda^{a_{2}},\left(a=\left(a_{1}, a_{2}\right) \in \mathbb{Z}^{(2)}\right.
$$

generate the basis in $\operatorname{gl}(N, \mathbb{C})$. We use the Greek letters for the elements of the lattice

$$
\tilde{\mathbb{Z}}_{N}^{(2)}=\mathbb{Z}_{N}^{(2)} \backslash(0,0) .
$$

Thus, $\left\{T_{\alpha}\right\}$ define a basis in $\operatorname{sl}(N, \mathbb{C})$. Since

$$
T_{a} T_{b}=\frac{1}{2 \pi \theta} \mathbf{e}_{\theta}\left(-\frac{a \times b}{2}\right) T_{a+b}, \quad\left(a \times b=a_{1} b_{2}-a_{2} b_{1}\right)
$$

the commutation relations in this basis assume the form

$$
\left[T_{\alpha}, T_{\beta}\right]=\mathbf{C}_{\theta}(\alpha, \beta) T_{\alpha+\beta},
$$

where

$$
\mathbf{C}_{\theta}(\alpha, \beta)=\frac{1}{\pi \theta} \sin \pi \theta(\alpha \times \beta)
$$

Let

$$
\mathbb{Z}_{\theta}^{(2)}(\tau)=\left(\gamma_{1}+\gamma_{2} \tau\right) \theta, \quad \gamma \in \tilde{\mathbb{Z}}_{N}^{(2)}
$$

be a regular lattice of order $N^{2}-1$ on $E_{\tau}$. Introduce the following constants on $\mathbb{Z}_{\theta}^{(2)}(\tau)$ :

$$
\begin{gathered}
\vartheta_{\theta}(\gamma)=\vartheta\left(\left(\gamma_{1}+\gamma_{2} \tau\right) \theta\right) \\
\zeta_{\theta}(\gamma)=\zeta\left(\left(\gamma_{1}+\gamma_{2} \tau\right) \theta\right), \quad \wp_{\theta}(\gamma)=\wp\left(\left(\gamma_{1}+\gamma_{2} \tau\right) \theta\right),
\end{gathered}
$$

and the quasi-periodic functions on $E_{\tau}$

$$
\begin{gathered}
\phi_{\theta}(\gamma, z)=\phi\left(\left(\gamma_{1}+\gamma_{2} \tau\right) \theta, z\right), \\
\varphi_{\theta}(\gamma, z)=\mathbf{e}_{\theta}\left(\gamma_{2} z\right) \phi_{\theta}(\gamma, z), \\
f_{\theta}(\gamma, z)=\left.\mathbf{e}_{\theta}\left(\gamma_{2} z\right) \partial_{u} \phi(u, z)\right|_{u=\left(\gamma_{1}+\gamma_{2} \tau\right) \theta} .
\end{gathered}
$$

It follows from (A.8) that

$$
\varphi_{\theta}(\gamma, z+1)=\mathbf{e}_{\theta}\left(\gamma_{2}\right) \varphi_{\theta}(\gamma, z), \quad \varphi_{\theta}(\gamma, z+\tau) \mathbf{e}_{\theta}\left(-\gamma_{1}\right) \varphi_{\theta}(\gamma, z) .
$$

\subsection{Appendix C. Non-commutative torus}

\section{Definition and representation.}

The non-commutative torus $\mathcal{A}_{\theta}$ is a unital algebra with the two generators $\left(U_{1}, U_{2}\right)$ that satisfy the relation

$$
U_{1} U_{2}=\mathbf{e}_{\theta}^{-1} U_{2} U_{1}, \mathbf{e}_{\theta}=e^{2 \pi i \theta}, \theta \in[0,1) .
$$

Elements of $\mathcal{A}_{\theta}$ are the double sums

$$
\mathcal{A}_{\theta}=\left\{X=\sum_{a_{1}, a_{2} \in \mathbb{Z}} c_{a_{1}, a_{2}} U_{1}^{a_{1}} U_{2}^{a_{2}} \mid c_{a_{1}, a_{2}} \in \mathbb{C}\right\},
$$


where $c_{a_{1}, a_{2}}$ are elements of the ring $\mathfrak{S}$ of rapidly decreasing sequences on $\mathbb{Z}^{2}$

$$
\mathfrak{S}=\left\{\left.c_{a_{1}, a_{2}}\left|\sup _{a_{1}, a_{2} \in \mathbb{Z}}\left(1+a_{1}^{2}+a_{2}^{2}\right)^{k}\right| c_{a_{1}, a_{2}}\right|^{2}<\infty \text { forall } k \in \mathbb{N}\right\} .
$$

The trace functional $\operatorname{tr}(x)$ on $\mathcal{A}_{\theta}$ is defined by

$$
\operatorname{tr}(X)=c_{00}
$$

It satisfies the evident identities

$$
\operatorname{tr}(1)=1, \quad \operatorname{tr}(X Y)=\operatorname{tr}(Y X)
$$

The dual space to $\mathfrak{S}$ is the space

$$
\mathfrak{S}^{\prime}=\left\{S_{a_{1}, a_{2}} \mid \sum_{a_{1}, a_{2}} c_{a_{1}, a_{2}} S_{-a_{1},-a_{2}}<\infty, c_{a_{1}, a_{2}} \in \mathfrak{S}\right\} .
$$

The relation with the commutative algebra of smooth functions on the two-dimensional torus

$$
T^{2}=\left\{\mathbb{R}^{2} / \mathbb{Z} \oplus \mathbb{Z}\right\} \sim\left\{0<x_{1} \leq 1,0<x_{2} \leq 1\right\} .
$$

comes from the identification

$$
U_{1} \rightarrow \mathbf{e}(x), \quad U_{2} \rightarrow \mathbf{e}(y)
$$

while the multiplication on $T^{2}$ becomes the Moyal multiplication:

$$
(f * g)(x):=f g+\sum_{n=1}^{\infty} \frac{(\pi \theta)^{n}}{n !} \varepsilon_{r_{1} s_{1}} \ldots \varepsilon_{r_{n} s_{n}}\left(\partial_{r_{1} \ldots r_{n}}^{n} f\right)\left(\partial_{s_{1} \ldots s_{n}}^{n} g\right) .
$$

The trace functional (C.3) in the Moyal identification is the integral

$$
\operatorname{tr} f=-\frac{1}{4 \pi^{2}} \int_{\mathcal{A}_{\theta}} f d x_{1} d x_{2}=f_{00} .
$$

We can identify $U_{1}, U_{2}$ with matrices from $\mathrm{GL}(\infty)$. Define $\mathrm{GL}(\infty)$ as the associative algebra of infinite matrices $c_{j k} E_{j k}$, where $E_{j k}=\left\|\delta_{j k}\right\|$, such that

$$
\sup _{j, k \in \mathbb{Z}}\left|c_{j k}\right|^{2}|j-k|^{n}<\infty \text { for } n \in \mathbb{N} .
$$

Consider the following two matrices from $\operatorname{GL}(\infty)$ :

$$
Q=\operatorname{diag}(\mathbf{e}(j \theta)) \text { and } \Lambda=\left\|\delta_{j, j+1}\right\|, j \in \mathbb{Z} .
$$

We have the following identification

$$
U_{1} \rightarrow Q, U_{2} \rightarrow \Lambda
$$

Another useful realization of $\mathcal{A}_{\theta}$ in the Schwartz space on $\mathbb{R}$ by the operators

$$
U_{1} f(x)=f(x-\theta), \quad U_{2} f(x)=\exp (2 \pi i x) f(x) .
$$

2. sin-algebra.

Define the following quadratic combinations of the generators

$$
T_{\alpha}=\frac{i}{2 \pi \theta} \mathbf{e}\left(\frac{\alpha_{1} \alpha_{2}}{2} \theta\right) U_{1}^{m} U_{2}^{n} \quad \alpha \in \tilde{\mathbb{Z}}^{(2)},
$$




$$
\tilde{\mathbb{Z}}^{(2)}=\left\{\alpha=\left(\alpha_{1}, \alpha_{2}\right), \alpha_{j} \in \mathbb{Z}, \alpha \neq(0,0)\right\} .
$$

Their commutator has the form of the sin-algebra

$$
\left[T_{\alpha}, T_{\beta}\right]=\mathbf{C}_{\theta}(\alpha, \beta) T_{\alpha+\beta}
$$

where

$$
\mathbf{C}_{\theta}(\alpha, \beta)=\frac{1}{\pi \theta} \sin \pi \theta(\alpha \times \beta) .
$$

We denote by $\sin _{\theta}$ the Lie algebra with the generators $T_{\alpha}$ over the ring $\mathfrak{S}$ (C.2)

$$
\psi=\sum_{\alpha} \psi_{\alpha} T_{\alpha}, \quad \psi_{\alpha} \in \mathfrak{S}
$$

and by $S I N_{\theta}$ the group of invertible elements from $\mathcal{A}_{\theta}$. The coalgebra $\sin _{\theta}^{*}$ is the linear space

$$
\sin _{\theta}^{*}=\left\{\mathbf{S}=\sum_{\alpha \in \tilde{\mathbb{Z}}^{(2)}} s_{\alpha} T \alpha, s_{\alpha} \in \mathfrak{S}^{\prime}\right\} .
$$

In the Moyal representation (C.7) the commutator of $\sin _{\theta}$ has the form

$$
[f(x, y), g(x, y)]=\{f, g\}^{\theta}:=\frac{1}{\theta}(f * g-g * f)
$$

\section{Elliptic constants related to $N C T \mathcal{A}_{\theta}$}

Introduce two numbers $\epsilon=\left(\epsilon_{1}, \epsilon_{2}\right)$ such that $\epsilon_{a} \theta<1$ and $\epsilon_{a} \theta$ are irrational. Consider the dense set $\mathbb{Z}_{\theta, \epsilon}(\tau)$ in $E_{\tau}$ :

$$
\mathbb{Z}_{\theta, \epsilon}(\tau)=\left\{\left(\epsilon_{1} \gamma_{1}+\tau \epsilon_{2} \gamma_{2}\right) \theta \in E_{\tau} \quad\left(\gamma_{1}, \gamma_{2}\right) \in \tilde{\mathbb{Z}}^{(2)}\right\} .
$$

The corresponding elliptic functions with the arguments from $\mathbb{Z}_{\theta, \epsilon}(\tau)$ are as follows:

$$
\begin{gathered}
\vartheta_{\theta, \epsilon}(\gamma)=\vartheta\left(\left(\epsilon_{1} \gamma_{1}+\epsilon_{2} \gamma_{2} \tau\right) \theta\right) \\
\zeta_{\theta, \epsilon}(\gamma)=\zeta\left(\left(\epsilon_{1} \gamma_{1}+\tau \epsilon_{2} \gamma_{2}\right) \theta\right), \quad \wp_{\theta, \epsilon}(\gamma)=\wp\left(\left(\epsilon_{1} \gamma_{1}+\tau \epsilon_{2} \gamma_{2}\right) \theta\right) \\
\phi_{\theta, \epsilon}(\gamma, z)=\phi\left(-\left(\epsilon_{1} \gamma_{1}+\tau \epsilon_{2} \gamma_{2}\right) \theta, z\right) \\
\varphi_{\theta, \epsilon}(\gamma)=\mathbf{e}_{\theta}\left(\epsilon_{2} \gamma_{2} z\right) \phi_{\theta, \epsilon}(\gamma, z) \\
f_{\theta, \epsilon}(\gamma)=\left.\mathbf{e}_{\theta}\left(\epsilon_{2} \gamma_{2} z\right) \partial_{u} \phi(u, z)\right|_{u=\left(\epsilon_{1} \gamma_{1}+\tau \epsilon_{2} \gamma_{2}\right) \theta}
\end{gathered}
$$

\section{Dispersionless limit.}

In the limit $\theta \rightarrow 0$ the Lie algebra $\sin _{\theta}$ becomes the Lie algebra of Hamiltonian functions

$$
\operatorname{Ham}\left(T^{2}\right) \sim C^{\infty}\left(T^{2}\right) / \mathbb{C}
$$

equipped with the canonical Poisson brackets. In $\operatorname{Ham}\left(T^{2}\right)$ we have the Fourier basis

$$
\mathbf{e}(\alpha \cdot x)=\exp \left(2 \pi i\left(\alpha_{1} x_{1}+\alpha_{2} x_{2}\right)\right)
$$

instead of the basis (C.11). The commutator (C.12) becomes

$$
[\mathbf{e}(\alpha x), \mathbf{e}(\beta x)]=(\alpha \times \beta) \mathbf{e}((\alpha+\beta) \cdot x) .
$$


The algebra $\operatorname{Ham}^{2} T^{2}$ ) (without constant Hamiltonians) is isomorphic to Lie algebra $S V e c t_{0}\left(T^{2}\right.$ ) of the divergence-free zero-flux vector fields on $T^{2}$ equipped with the area form $-4 \pi^{2} d x_{1} d x_{2}$. Let $h\left(x_{1}, x_{2}\right) \in \operatorname{Ham}\left(T^{2}\right)$. Then the Hamiltonian field $V_{h}$ corresponding to the Hamiltonian function $h$ is

$$
V_{h}=-\frac{1}{4 \pi^{2}}\left(\left(\partial_{2} h\right) \partial_{1}-\left(\partial_{1} h\right) \partial_{2}\right)
$$

while

$$
\left[V_{h}, V_{h^{\prime}}\right]=V_{\left\{h, h^{\prime}\right\}}
$$

For $f(x)=\sum_{\alpha} f_{\alpha} \mathbf{e}(\alpha \cdot x)$

$$
\int_{T^{2}} f=-\frac{1}{4 \pi^{2}} f_{0}
$$

\section{References}

[1] S. Ziglin, Non-integrability of the problem of the motion of four point vortices, Dokl. Akad. Nauk SSSR 250, no. 6, (1980) 1296-1300.

[2] A.G. Reyman and M.A. Semenov-Tyan-Shanskii, Lie algebras and Lax equations with spectral parameter on elliptic curve, Notes of the LOMI Seminar, Vol. 150, (1986), 104-118.

[3] A. Levin, M. Olshanetsky and A. Zotov, Hitchin Systems: symplectic maps and twodimensional version, [nlin.SI/0110045].

[4] A. Belavin and V. Drinfeld, Solutions of the classical Yang-Baxter equation for simple Lie algebras, Funct. Anal and Applic., 16 (1982), no. 3, 1-29.

[5] B. Feigin and A. Odesski, Sklyanin's elliptic algebras, Funct. Anal. and Applic., 23 (1989), no. $3,207-214$.

[6] V.I. Arnold, The Hamiltonian nature of the Euler equation in the dynamics of rigid body and of an ideal fluid, Russ. Math. Surveys, 24 (1969), no. 3, 225-226.

[7] V.I. Arnold and B.A. Khesin, Topological methods in Hydrodynamics, Applied Mathematical Science, Vol. 125, Springer-Verlag, New York, (1998).

[8] S. Manakov, A remark on the integration of the Eulerian equations of the dynamics of an n-dimensional rigid body, Funct. Anal. and Applic., 10 (1976), no. 4, 93-94.

[9] R.S. Ward, Infinite-dimensional gauge groups and special nonlinear gravitons, J.Geom. and Phys. 8 (1992), no.1-4, 317-325.

[10] B. Khesin and V. Ovsienko, The super Korteweg-de Vries equation as an Euler equation, Funct. Anal. and Applic. 21 (1987), no. 4, 81-82.

[11] G. Segal, The geometry of the KdV equation. Topological methods in quantum field theory, (Trieste, 1990). Internat. J. Modern Phys. A6 (1991), no. 16, 2859-2869.

[12] B. Khesin and G. Misiolek Euler equations on homogeneous spaces and virasoro orbits, Advances in Math. 176 (2003), 116-144.

[13] A. Belavin, Discrete groups and integrability of quantum systems, Funct. Anal. and Applic., 14 (1980), 18-26. 
[14] E.K. Sklyanin, Some algebraic structures connected with the Yang-Baxter equation, Funct. Anal. and Applic., 16 (1982), no. 4, 27-34,

[15] M. Olshanetsky, Integrable tops and non-commutative torus, talk at the XVI Max Born simposium "Supersymmetries and Quantum Symmetries", ed. E.Ivanov et al, Dubna 2002.

[16] P.I. Etingof and I.B. Frenkel, Central extensions of current groups in two dimensions, Commun. Math. Phys. 165 (1994), no.3, 429-444.

[17] M. Semenov-Tyan-Shansky, What a classical r-matrix is, Funct. Anal. and Applic., 17 (1983), no. 4, 17-33.

[18] H.W. Braden, V.A. Dolgushev, M.A. Olshanetsky, and A.V. Zotov, Classical R-Matrices and the Feigin-Odesskii Algebra via Hamiltonian and Poisson Reductions, [hep-th/0301121]

[19] F. Magri, A simple model of the integrable Hamiltonian equation, J. Math. Phys., 19 (1978), $1156-1162$. 